\title{
Inductive equivalence in clausal logic and nonmonotonic logic programming
}

\author{
Chiaki Sakama $\cdot$ Katsumi Inoue
}

Received: 24 September 2007 / Revised: 9 July 2009 / Accepted: 27 April 2010 /

Published online: 20 May 2010

(C) The Author(s) 2010

\begin{abstract}
This paper provides a logical framework for comparing inductive capabilities among agents having different background theories. A background theory is called inductively equivalent to another background theory if the two theories induce the same hypotheses for any observation. Conditions of inductive equivalence change depending on the logic of representation languages and the logic of induction or inductive logic programming (ILP). In this paper, we consider clausal logic and nonmonotonic logic programs as representation languages for background theories. Then we investigate conditions of inductive equivalence in four different frameworks of induction, cautious induction, brave induction, learning from satisfiability, and descriptive induction. We observe that several induction algorithms in Horn ILP systems require weaker conditions of equivalence under restricted problem settings. We address that inductive equivalence can be used for verification and evaluation of induction algorithms, and argue problems for optimizing background theories in ILP.
\end{abstract}

Keywords Inductive equivalence $\cdot$ Inductive logic programming $\cdot$ Nonmonotonic logic programs

\section{Introduction}

Equivalence relations between logical theories have been studied in many ways in artificial intelligence and logic programming. In knowledge representation, a theory represents knowledge of a problem domain. The same problem would be represented in different ways by different experts. Equivalence of two theories is then used for evaluating information

Editor: David Page.

C. Sakama (凶)

Department of Computer and Communication Sciences, Wakayama University, Sakaedani, Wakayama, 640-8510, Japan

e-mail: sakama@sys.wakayama-u.ac.jp

K. Inoue

National Institute of Informatics, 2-1-2 Hitotsubashi, Chiyoda-ku, Tokyo, 101-8430, Japan

e-mail: ki@nii.ac.jp 
content and identifying different information sources. In program development, one program may give a declarative specification of some problem and another program may give an efficient coding of it. In this case, equivalence of two programs guarantees a correct implementation of the given specification. In the context of logic programming, various criteria for equivalence relations are proposed in the literature (Maher 1988; Sagiv 1988; Lifschitz et al. 2001; Eiter and Fink 2003; Inoue and Sakama 2004). Among them, weak equivalence and strong equivalence of two programs are particularly important. Two logic programs $P_{1}$ and $P_{2}$ are (weakly) equivalent if they have the same declarative meaning. On the other hand, two programs $P_{1}$ and $P_{2}$ are strongly equivalent if they preserve the equivalence relation by the introduction of arbitrary rules $R$ to them.

Equivalence relations presented above compare capabilities of deductive reasoning between programs. For instance, two Horn logic programs are weakly equivalent if they have the same least model that is the logical consequences of each program. When we consider realizing intelligent agents that can perform commonsense reasoning, however, comparing capabilities of non-deductive reasoning between programs is also necessary and important. Recently, Inoue and Sakama (2005, 2006a, 2006b) argue equivalence in $a b$ ductive logic. They introduce two different types of abductive equivalence: explainable equivalence and explanatory equivalence. The former considers whether two theories have the same explainability for any observation, while the latter considers whether two theories have the same explanation contents for any observation. These two notions compare capabilities of abductive reasoning among agents, and they provide necessary and sufficient conditions for abductive equivalence in first-order logic and abductive logic programming (ALP) (Denecker and Kakas 2002). Induction is also known as non-deductive reasoning, which is often distinguished from abduction (Flach and Kakas 2000). In computational logic, induction is realized by inductive logic programming (ILP) (Muggleton 1992; Nienhuys-Cheng and De Wolf 1997). A typical induction problem is to build a hypothesis which covers a given observation with respect to a background theory. Then, there are some questions concerning equivalence issues in induction.

1. When can we say that induction with a background theory is equivalent to induction with another background theory?

Two different background theories $B_{1}$ and $B_{2}$ are considered equivalent if they induce the same hypothesis $H$ for any observation $O$. This equivalence measure is useful for comparing "information contents" of different background theories.

2. When can we say that induced hypotheses are equivalent to another induced hypotheses? Two hypotheses $H_{1}$ and $H_{2}$ are considered equivalent if they account for the same observation $O$ with respect to a background theory $B$. This equivalence measure is useful for comparing "explanation power" of different hypotheses.

3. When can we say that induction from an observation is equivalent to induction from another observation?

Two observations $O_{1}$ and $O_{2}$ are considered equivalent if they produce the same hypothesis $H$ with respect to a background theory $B$. This equivalence measure is useful for comparing "evidential power" of different observations.

4. Do conditions for these equivalence differ by underlying logics?

The results of induction and equivalence conditions generally depend on a logic on which induction is based. Moreover, those conditions differ among individual induction algorithms. Then, we can compare different logics or algorithms for estimating their induction capabilities.

These issues are important and meaningful for comparing different induction tasks, but few studies have argued the problems so far. In this paper, we focus on the question (1) above 
and study the problem of equivalence of background theories in induction. To answer the question (4), we also investigate conditions of equivalence in different logics and induction algorithms. Other problems, concerning the questions (2) and (3), are studied in a different paper (Sakama and Inoue 2009a).

To formalize the problem, we introduce the notion of inductive equivalence between background theories. A background theory $B_{1}$ is said inductively equivalent to another background theory $B_{2}$ if $B_{1}$ and $B_{2}$ induce the same hypothesis $H$ for an arbitrary observation $O$. Intuitively, if an agent has a background theory $B_{1}$ which is inductively equivalent to another background theory $B_{2}$ of another agent, then these two agents are considered equivalent with respect to inductive capability. In this case, we can identify those two agents as far as induction is concerned. From the viewpoint of program development, if a theory $B_{1}$ is transformed to another syntactically different $B_{2}$, inductive equivalence of two theories guarantees identification of results of induction from each theory. This provides guidelines for optimizing background theories in ILP. The problem of interest is logical conditions for inductive equivalence in ILP. Conditions for inductive equivalence differ depending on logics of representation languages and logics of induction. This paper considers two logics for representation languages - clausal logic and nonmonotonic logic programming. These logics are widely used in knowledge representation and ILP (Muggleton 1992; Baral and Gelfond 1994). On the other hand, we consider four different frameworks of induction, cautious induction, brave induction (Sakama and Inoue 2009b), learning from satisfiability (De Raedt 1997; De Raedt and Dehaspe 1997a), and descriptive induction (Lachiche 2000). These frameworks capture different aspects of induction problems. We show necessary and sufficient conditions for inductive equivalence under different semantics in respective induction. We also observe that some induction algorithms in Horn ILP systems require weaker conditions of inductive equivalence. We address that inductive equivalence is used for testing correctness/completeness of an induction algorithm and comparing capabilities of different algorithms. We also argue problems for optimizing background theories in ILP through appropriate program transformations.

This paper is a revised and extended version of (Sakama and Inoue 2005). The differences between the present work and the previous one are follows. First, we apply the framework of inductive equivalence to different types of induction, and investigate formal properties among them. Inductive equivalences for cautious induction, brave induction, and learning from satisfiability are new in this paper. Second, inductive equivalences in particular induction algorithms are also revised and extended. Inductive equivalences in FOIL and BRAIN ${ }^{n o t}$ are new in this paper. Third, previous results for inductive equivalence in nonmonotonic logic programs are generalized to background theories which possibly contain disjunctions. Fourth, new considerations and additional arguments are added throughout the paper.

The rest of this paper is organized as follows. Section 2 presents logical frameworks used in this paper. Section 3 introduces the notion of inductive equivalence and investigates formal properties in clausal logic. Section 4 verifies conditions of inductive equivalence in some Horn ILP systems. Section 5 applies inductive equivalence to nonmonotonic logic programs. Section 6 discusses related issues and potential applications. Finally, Section 7 concludes the paper.

\section{Logical framework}

\subsection{Clausal theories}

A first-order language consists of an alphabet and all formulas defined over it. The definition is the standard one in the literature (Nienhuys-Cheng and De Wolf 1997, for instance). 
A first-order theory is a set of formulas. A clause is a formula of the form

$$
A_{1} \vee \cdots \vee A_{m} \vee \neg A_{m+1} \vee \cdots \vee \neg A_{n}
$$

where $A_{i}(1 \leq i \leq n)$ are atoms and every variable appearing in (1) is universally quantified at the front. A clausal theory (or simply a theory) is a set of clauses. The clause (1) is also written as

$$
A_{1} \vee \cdots \vee A_{m} \leftarrow A_{m+1}, \ldots, A_{n}
$$

in the context of logic programming. The disjunction $A_{1} \vee \cdots \vee A_{m}$ is the head and the conjunction $A_{m+1}, \ldots, A_{n}$ is the body of the clause. In particular, a clause (2) having at most one atom in its head is a Horn clause and a set of Horn clauses is a Horn logic program. A Horn clause is called a definite clause if it contains exactly one atom in its head. A definite logic program is a set of definite clauses. A clause $A \leftarrow$ is called a fact and is identified with the atom $A$. A theory is identified with the conjunction of clauses included in the theory. A theory, a clause or an atom is ground if it contains no variable. A theory or a clause with variables is identified with the set of its ground instances. A propositional theory is a finite set of ground clauses. Clausal theories and Horn logic programs are subsets of first-order theories, while nonmonotonic logic programs, which are handled in Sect. 4, are outside of first-order logic.

The domain of a theory is given as the Herbrand universe and interpretations are defined as subsets of the Herbrand base $H B$. An interpretation $M$ satisfies the ground clause of the form (2) if $\left\{A_{m+1}, \ldots, A_{n}\right\} \subseteq I$ implies $\left\{A_{1}, \ldots, A_{m}\right\} \cap I \neq \emptyset$. M satisfies a theory $T$ if $M$ satisfies every ground instance of any clause in $T$. An interpretation $M$ is a model of a theory $T$ if $M$ satisfies $T$. The set of all models of $T$ is written as $\operatorname{Mod}(T)$. The semantics of a theory $T$ is represented as a subset $\operatorname{SEM}(T)$ of $\operatorname{Mod}(T)$, i.e.,

$$
\operatorname{SEM}(T) \subseteq \operatorname{Mod}(T) .
$$

The set $\operatorname{SEM}(T)$ represents models that are selected from $\operatorname{Mod}(T)$ based on some preference criterion. In particular, $\operatorname{SEM}(T)=\operatorname{Mod}(T)$ holds under the classical model theory of first-order logic. A theory $T$ is consistent under $\operatorname{SEM}$ if $\operatorname{SEM}(T) \neq \emptyset$; otherwise, $T$ is inconsistent. A theory $T$ satisfies a clause $C$ (written as $T \models C$ ) if $C$ is satisfied in every model of $T$. $T$ satisfies a set $S$ of clauses (written as $T \models S$ ) if $T \models C$ for any clause $C$ in $S$. There are several criteria for selecting models as $\operatorname{SEM}(T)$. Among them, minimal models are often considered in the literature. The set of all minimal models of $T$ (denoted by $M M(T)$ ) is defined as

$$
M M(T)=\{M \in \operatorname{Mod}(T) \mid \neg \exists N \in \operatorname{Mod}(T) \text { such that } N \subset M\} .
$$

Every consistent clausal theory has a minimal model (Bossu and Siegel 1985).

\subsection{Logics of induction}

There are several definitions of induction. In this paper, we consider the following four different frameworks of induction:

- Cautious induction (Sakama and Inoue 2009b)

- Brave induction (Sakama and Inoue 2009b)

- Learning from satisfiability (De Raedt 1997; De Raedt and Dehaspe 1997a)

- Descriptive induction (Lachiche 2000) 
Let $B, H$, and $O$ be sets of formulas respectively representing a background theory, a hypothesis, and an observation. Then, each induction is defined as follows. ${ }^{1}$

Cautious induction: Given $B$ and $O$, find $H$ such that $O$ is satisfied by every $M \in$ $\operatorname{SEM}(B \cup H)$ where $B \cup H$ is consistent.

Brave induction: Given $B$ and $O$, find $H$ such that $O$ is satisfied by some $M \in$ $\operatorname{SEM}(B \cup H)$ where $B \cup H$ is consistent.

Learning from satisfiability: Given $B$ and $O$, find $H$ such that $B \cup H \cup O$ is consistent under $S E M$.

Descriptive induction: Give $B$ and $O$, find $H$ such that $H$ is satisfied by every $M \in$ $\operatorname{SEM}(B \cup O)$ where $B \cup O$ is consistent.

In each case, we say that a hypothesis $H$ covers (or explains) $O$ with respect to $B$ (under $S E M$ ) in the induction framework $I . H$ is also called a solution in $I$. Here, $I$ is one of the four induction frameworks presented above. In this paper, cautious induction, brave induction, learning from satisfiability, and descriptive induction is respectively abbreviated as Caulnd, Bralnd, LFS, and DesInd.

Cautious induction requires an observation to be satisfied in every model in $\operatorname{SEM}(B \cup H)$. In particular, when $\operatorname{SEM}(B \cup H)=\operatorname{Mod}(B \cup H)$ in first-order logic, it is written as

$$
B \cup H \models E .
$$

In this case, cautious induction is also called explanatory induction (abbreviated as Explnd) (Flach 1996), which is known as usual setting in ILP (Muggleton 1992; Nienhuys-Cheng and De Wolf 1997). Brave induction, on the other hand, requires that an observation is satisfied in some models in $\operatorname{SEM}(B \cup H)$. By the definition, brave induction is weaker than cautious induction, that is, if $H$ is a solution of cautious induction, it is also a solution of brave induction, but not vice versa. Learning from satisfiability is weaker than brave induction, so that it provides the weakest form of induction among those three frameworks. Brave induction and learning from satisfiability coincide when $\operatorname{SEM}(B)=\operatorname{Mod}(B)$. That is, $O$ is satisfied in some model of $B \cup H$ iff $B \cup H \cup O$ is consistent. Descriptive induction, which is also called confirmatory induction (Flach 1996), prescribes that a hypothesis is satisfied in a background theory and an observation. In contrast to explanatory induction, it does not intend to learn classification rules but seek regularities over observed data. When an observation is given as a set of interpretations, descriptive induction is also called learning from interpretations (De Raedt 1997; De Raedt and Dehaspe 1997b).

Example 2.1 (Sakama and Inoue 2009b) Suppose that there are 30 students in a class, of which 20 are European, 7 are Asian, and 3 are American. The situation is represented by the background theory $B$ and the observation $O$ :

$$
\begin{aligned}
& B=\{\text { student }(1), \ldots, \text { student }(30)\} \\
& O=\{\operatorname{euro}(1), \ldots, \operatorname{euro}(20), \operatorname{asia}(21), \ldots, \operatorname{asia}(27), \operatorname{usa}(28), \ldots, u s a(30)\}
\end{aligned}
$$

where each number represents individual students. Put the semantics of the background theory as the minimal model semantics, $\operatorname{SEM}(B)=M M(B)$. First, consider the set $H_{1}$ of

\footnotetext{
${ }^{1}$ Observations defined here are positive observations. In the literature, negative observations are often considered as well as positive ones. For simplicity reasons, we consider only positive observations in this paper.
} 
the following clauses

$$
\begin{aligned}
& \text { euro }(x) \leftarrow \operatorname{student}(x), \\
& \operatorname{asia}(x) \leftarrow \operatorname{student}(x), \\
& u \operatorname{sa}(x) \leftarrow \operatorname{student}(x) .
\end{aligned}
$$

Then, $H_{1}$ is a solution of Bralnd, Caulnd, and LFS, but it is not a solution of Desind.

Next, consider the set $\mathrm{H}_{2}$ which consists of the single clause

$$
\operatorname{euro}(x) \vee \operatorname{asia}(x) \vee u s a(x) \leftarrow \operatorname{student}(x)
$$

$\mathrm{H}_{2}$ is a solution of Bralnd, LFS, and DesInd, but it is not a solution of Caulnd.

Finally, consider the set $\mathrm{H}_{3}$ which consists of the single clause

$$
\operatorname{student}(x) \leftarrow \operatorname{euro}(x)
$$

$\mathrm{H}_{3}$ is a solution of DesInd and LFS, but it is not a solution of Bralnd nor Caulnd.

Thus, four induction frameworks provide different solutions in general.

\subsection{Equivalence relation}

Two different theories are equivalent in many ways. In this paper, we handle three different notions of equivalences. Consider two theories $T_{1}$ and $T_{2}$ which have the common underlying language. Then, $T_{1}$ and $T_{2}$ are

- logically equivalent (written as $\left.T_{1} \equiv T_{2}\right)$ if $\operatorname{Mod}\left(T_{1}\right)=\operatorname{Mod}\left(T_{2}\right)$.

- weakly equivalent (written as $T_{1} \equiv_{w} T_{2}$ ) if $\operatorname{SEM}\left(T_{1}\right)=\operatorname{SEM}\left(T_{2}\right)$.

- strongly equivalent (written as $T_{1} \equiv_{s} T_{2}$ ) if $T_{1} \cup U \equiv_{w} T_{2} \cup U$ for any theory $U$ under the same language.

By the definition, $T_{1} \equiv_{s} T_{2}$ implies $T_{1} \equiv_{w} T_{2}$. In particular, three equivalence relations coincide in first-order logic under the condition $\operatorname{SEM}(T)=\operatorname{Mod}(T)$ (Eiter and Fink 2003).

We first show that logical equivalence coincides with strong equivalence when $\operatorname{SEM}(T)=M M(T)$ in clausal logic.

Proposition 2.1 Let $T$ be a clausal theory and $H B$ its Herbrand base. For any $M(\subseteq H B)$, put $M^{*}=M \cup\{\neg A \mid A \in H B \backslash M\}$. Then, $M$ is a model of a theory $T$ iff $T \cup M^{*}$ is consistent.

Proof If $M$ is a model of $T$, so is $M^{*}$. Then, $T \cup M^{*}$ is consistent. Conversely, when $T \cup M^{*}$ is consistent, assume that $M$ is not a model of $T$. Then, there is a clause $C$ in $T$ which is not satisfied by $M$. In this case, $M^{*} \cup\{C\}$ is inconsistent. This contradicts the fact that $T \cup M^{*}$ is consistent.

Proposition 2.2 (Logical equivalence vs. strong equivalence under MM) Let $T_{1}$ and $T_{2}$ be two clausal theories. Then, $T_{1} \equiv T_{2}$ iff $M M\left(T_{1} \cup U\right)=M M\left(T_{2} \cup U\right)$ for any clausal theory $U$.

Proof The only-if part is obvious. Assume $M M\left(T_{1} \cup U\right)=M M\left(T_{2} \cup U\right)$ for any $U$. If $T_{1} \not \equiv T_{2}$, there is either $M \in \operatorname{Mod}\left(T_{1}\right) \backslash \operatorname{Mod}\left(T_{2}\right)$ or $M \in \operatorname{Mod}\left(T_{2}\right) \backslash \operatorname{Mod}\left(T_{1}\right)$. Consider 
the case $M \in \operatorname{Mod}\left(T_{1}\right) \backslash \operatorname{Mod}\left(T_{2}\right)$. Since $M$ is not a model of $T_{2}, T_{2} \cup M^{*}$ is inconsistent (Proposition 2.1). Thus, $M M\left(T_{2} \cup M^{*}\right)=\emptyset$. On the other hand, $M \in \operatorname{Mod}\left(T_{1}\right)$ implies $M \in \operatorname{Mod}\left(T_{1} \cup M^{*}\right)$. Since $T_{1} \cup M^{*}$ is a clausal theory, $M \in \operatorname{Mod}\left(T_{1} \cup M^{*}\right)$ implies the existence of minimal models. Hence, $M M\left(T_{1} \cup M^{*}\right) \neq \emptyset$. This contradicts the assumption. The case of $M \in \operatorname{Mod}\left(T_{2}\right) \backslash \operatorname{Mod}\left(T_{1}\right)$ is proved in the same manner. Hence, $T_{1} \equiv T_{2}$.

Proposition 2.3 (Logical equivalence vs. weak equivalence under MM) Let $T_{1}$ and $T_{2}$ be two clausal theories. Then, $T_{1} \equiv T_{2}$ implies $T_{1} \equiv_{w} T_{2}$ under the minimal model semantics.

The converse of Proposition 2.3 does not hold in general.

Example 2.2 Consider three clausal theories:

$$
\begin{aligned}
& T_{1}=\{a \vee b, \quad c \vee \neg a, \quad c \vee \neg b\}, \\
& T_{2}=\{a \vee b, \quad c\}, \\
& T_{3}=\{a \vee b, \quad \neg a \vee \neg b, \quad c\} .
\end{aligned}
$$

First, set $\operatorname{SEM}\left(T_{i}\right)=\operatorname{Mod}\left(T_{i}\right)$ for $i=1,2,3$. Then,

$$
\operatorname{Mod}\left(T_{1}\right)=\operatorname{Mod}\left(T_{2}\right)=\{\{a, c\},\{b, c\},\{a, b, c\}\} \quad \text { and } \operatorname{Mod}\left(T_{3}\right)=\{\{a, c\},\{b, c\}\} .
$$

In this case, the following relations hold: $T_{1} \equiv T_{2}, T_{1} \not \equiv T_{3}$ and $T_{2} \not \equiv T_{3}$.

Next, set $\operatorname{SEM}\left(T_{i}\right)=M M\left(T_{i}\right)$ for $i=1,2,3$. Then,

$$
M M\left(T_{1}\right)=M M\left(T_{2}\right)=M M\left(T_{3}\right)=\{\{a, c\},\{b, c\}\} .
$$

In this case, the following relations hold: $T_{1} \equiv_{w} T_{2} \equiv_{w} T_{3}, T_{1} \equiv_{s} T_{2}, T_{1} \not \equiv_{s} T_{3}$ and $T_{2} \not \equiv_{s} T_{3}$. Here, $T_{2} \not \equiv_{s} T_{3}$ because the addition of $Q=\{a, b\}$ makes $T_{3}$ inconsistent.

\section{Inductive equivalence in clausal logic}

\subsection{Inductive equivalence}

We first provide a general framework of inductive equivalence between two theories.

Definition 3.1 (Inductive equivalence) Let $B_{1}$ and $B_{2}$ be two background theories having the same Herbrand base $H B$. For any observation $O$, suppose that a hypothesis $H$ covers $O$ with respect to $B_{1}$ under SEM in induction $I$ iff $H$ covers $O$ with respect to $B_{2}$ under SEM in induction $I$. In this case, $B_{1}$ and $B_{2}$ are said to be inductively equivalent under SEM in $I$ (written $B_{1} \equiv{ }_{I}^{S E M} B_{2}$ ).

By the definition, inductive equivalence presents that two background theories have the same explanation contents for any observation. Note that there are at least three different parameters on which inductive equivalence depends-(i) syntax of $B, H$ and $O$, (ii) the underlying semantics $S E M$, (iii) and the framework of induction $I$. In this paper, we study several cases of inductive equivalence with different parameters.

The notion of inductive equivalence is applied to four induction frameworks as follows. 
Definition 3.2 (Inductive equivalence in different frameworks of induction) Let $B_{1}$ and $B_{2}$ be two theories having the same Herbrand base $H B$. Then,

1. $B_{1}$ and $B_{2}$ are inductively equivalent under SEM in cautious induction (written $\left.B_{1} \equiv{ }_{\text {Caulnd }}^{S E M} B_{2}\right)$ if for any $O$ and any $H, O$ is satisfied by every $M \in \operatorname{SEM}\left(B_{1} \cup H\right)$ iff $O$ is satisfied by every $M \in \operatorname{SEM}\left(B_{2} \cup H\right)$, where $B_{1} \cup H$ and $B_{2} \cup H$ are consistent.

2. $B_{1}$ and $B_{2}$ are inductively equivalent under $S E M$ in brave induction (written $\left.B_{1} \equiv{ }_{\text {Bralnd }}^{S E M} B_{2}\right)$ if for any $O$ and any $H, O$ is satisfied by some $M \in \operatorname{SEM}\left(B_{1} \cup H\right)$ iff $O$ is satisfied by some $M \in \operatorname{SEM}\left(B_{2} \cup H\right)$, where $B_{1} \cup H$ and $B_{2} \cup H$ are consistent.

3. $B_{1}$ and $B_{2}$ are inductively equivalent under SEM in learning from satisfiability (written $\left.B_{1} \equiv{ }_{\mathrm{LFS}}^{S E M} B_{2}\right)$ if for any $O$ and any $H, B_{1} \cup H \cup O$ is consistent iff $B_{2} \cup H \cup O$ is consistent.

4. $B_{1}$ and $B_{2}$ are inductively equivalent under SEM in descriptive induction (written $\left.B_{1} \equiv{ }_{\text {DesInd }}^{S E M} B_{2}\right)$ if for any $O$ and any $H, H$ is satisfied by every $M \in \operatorname{SEM}\left(B_{1} \cup O\right)$ iff $H$ is satisfied by every $M \in S E M\left(B_{2} \cup O\right)$, where $B_{1} \cup O$ and $B_{2} \cup O$ are consistent.

Proposition 3.1 (Relations between different inductive equivalences) For any SEM, the following relations hold.

1. $B_{1} \equiv \underset{\text { Caulnd }}{S E M} B_{2}$ implies $B_{1} \equiv \underset{\text { Bralnd }}{S E M} B_{2}$.

2. $B_{1} \equiv \underset{\text { Bralnd }}{\text { SEM }} B_{2}$ implies $B_{1} \equiv \underset{\mathrm{LFS}}{\mathrm{SEM}} B_{2}$.

3. $B_{1} \equiv \underset{\text { Caulnd }}{\operatorname{SEM}} B_{2}$ iff $B_{1} \equiv \underset{\text { Desind }}{\text { SEM }} B_{2}$.

Proof The results of (1) and (2) hold by their definition. To see (3), the definition of inductive equivalence in descriptive induction is obtained by exchanging the positions of $O$ and $H$ in the definition in cautious induction. Since both $O$ and $H$ are arbitrary sets of formulas, the result holds.

Proposition 3.1(1) and (2) show that the implication relations among three induction frameworks are inherited to equivalence relations. On the other hand, Proposition 3.1(3) represents that in the context of inductive equivalence, distinction between cautious induction and descriptive induction is unimportant. With this reason, we mainly consider inductive equivalence in cautious induction, brave induction, and learning from satisfiability, hereafter.

\subsection{Inductive equivalence between clausal theories}

In this section, we consider the following problem setting:

- a background theory $B$ is given as a clausal theory

- an observation $O$ is a set of clauses

- a hypothesis $H$ is a set of clauses

We first set $\operatorname{SEM}(B)=\operatorname{Mod}(B)$, i.e, the classical semantics in first-order logic. In this case, cautious induction coincides with explanatory induction, and brave induction coincides with learning from satisfiability, as presented in Sect. 2.2. Necessary and sufficient conditions for inductive equivalence are stated below.

Theorem 3.2 (Condition for inductive equivalence in explanatory induction) For any two clausal theories $B_{1}$ and $B_{2}, B_{1} \equiv{ }_{\text {Caulnd }}^{\text {Mod }} B_{2}$ iff $B_{1} \equiv B_{2}$. 
Proof Let $O$ and $H$ be arbitrary sets of clauses. Then, $B_{1}$ and $B_{2}$ are inductively equivalent in cautious induction

iff $B_{1} \cup H \models O \Leftrightarrow B_{2} \cup H \models O$ for any $O$ and $H$ such that $B_{1} \cup H$ and $B_{2} \cup H$ are consistent

iff $B_{1} \models H \rightarrow O \Leftrightarrow B_{2} \models H \rightarrow O$ for any $O$ and $H$ such that $B_{1} \cup H$ and $B_{2} \cup H$ are consistent

iff $B_{1} \equiv B_{2}$.

Theorem 3.3 (Condition for inductive equivalence in brave induction)

For any two clausal theories $B_{1}$ and $B_{2}, B_{1} \equiv{ }_{\text {Bralnd }}^{M o d} B_{2}$ iff $B_{1} \equiv B_{2}$.

Proof Let $O$ and $H$ be arbitrary sets of clauses. Then, $B_{1}$ and $B_{2}$ are inductively equivalent in brave induction

iff $B_{1} \cup H \cup O$ is consistent $\Leftrightarrow B_{1} \cup H \cup O$ is consistent for any $O$ and $H$

iff $B_{1} \cup O$ is consistent $\Leftrightarrow B_{1} \cup O$ is consistent for any $O$

iff $B_{1} \not \models \neg O \Leftrightarrow B_{2} \not \models \neg O$ for any $O$

iff $B_{1} \models F \Leftrightarrow B_{2} \models F$ for any formula $F$

iff $B_{1} \equiv B_{2}$.

By Theorems 3.2 and 3.3, the following result follows.

Corollary 3.4 (Inductive equivalence in cautious induction and brave induction) For any two clausal theories $B_{1}$ and $B_{2}, B_{1} \equiv \underset{\text { Caulnd }}{\text { Mod }} B_{2}$ iff $B_{1} \equiv \underset{\text { Bralnd }}{\text { Mod }} B_{2}$.

By the fact that descriptive induction is identified with cautious induction (Proposition 3.1), we conclude that the inductive equivalence relations in four different induction frameworks coincide under the classical semantics.

Next, we set $\operatorname{SEM}(B)=M M(B)$ for the semantics of a clausal theory $B$. This setting is considered as the minimal model semantics of disjunctive logic programs (Minker 1982) or circumscription (McCarthy 1980). In this case, we have the next result.

Theorem 3.5 (Condition for inductive equivalence under MM in cautious induction) For any two clausal theories $B_{1}$ and $B_{2}, B_{1} \equiv{ }_{\text {Caulnd }}^{M M} B_{2}$ iff $B_{1} \equiv B_{2}$.

Proof Suppose that $B_{1}$ and $B_{2}$ are inductively equivalent under the minimal model semantics in Caulnd. Then, for any set $O$ and for any set $H$ of clauses, $O$ is satisfied by any $M \in M M\left(B_{1} \cup H\right)$ iff $O$ is satisfied by any $N \in M M\left(B_{2} \cup H\right)$ where $B_{1} \cup H$ and $B_{2} \cup H$ are consistent. By putting $O=B_{1} \cup H$, it holds that $B_{1} \cup H$ is satisfied by any $M \in M M\left(B_{1} \cup H\right)$ iff $B_{1} \cup H$ is satisfied by any $N \in M M\left(B_{2} \cup H\right)$. By putting $O=B_{2} \cup H$, it holds that $B_{2} \cup H$ is satisfied by any $M \in M M\left(B_{1} \cup H\right)$ iff $B_{2} \cup H$ is satisfied by any $N \in M M\left(B_{2} \cup H\right)$. As $B_{1} \cup H$ is satisfied by any $M \in M M\left(B_{1} \cup H\right)$ and $B_{2} \cup H$ is satisfied by any $N \in M M\left(B_{2} \cup H\right)$, it holds that $B_{1} \cup H$ is satisfied by any $N \in M M\left(B_{2} \cup H\right)$ and $B_{2} \cup H$ is satisfied by any $M \in M M\left(B_{1} \cup H\right)$. Since any minimal model $M$ of $B_{1} \cup H$ satisfies every clause in $B_{2} \cup H, M \in \operatorname{Mod}\left(B_{2} \cup H\right)$. If $M \notin M M\left(B_{2} \cup H\right)$, there is a minimal model $I \in M M\left(B_{2} \cup H\right)$ such that $I \subset M$ and $I$ satisfies $B_{2} \cup H$. Since any minimal model $I$ of $B_{2} \cup H$ satisfies every clause in $B_{1} \cup H, I \in \operatorname{Mod}\left(B_{1} \cup H\right)$. But this is impossible because $M$ is a minimal model of $B_{1} \cup H$. Hence, $M \in M M\left(B_{2} \cup H\right)$. Likewise, $N \in M M\left(B_{2} \cup H\right)$ implies $N \in M M\left(B_{1} \cup H\right)$. Therefore, $M M\left(B_{1} \cup H\right)=M M\left(B_{2} \cup H\right)$, so that $B_{1} \equiv B_{2}$ by Proposition 2.2. 
Conversely, if $B_{1} \equiv B_{2}, M M\left(B_{1} \cup H\right)=M M\left(B_{2} \cup H\right)$ holds for any set $H$ of clauses (Proposition 2.2). Then, for any set $H$ and for any set $O$ of clauses, $O$ is satisfied by any $M \in M M\left(B_{1} \cup H\right)$ iff $O$ is satisfied by any $N \in M M\left(B_{2} \cup H\right)$, and $B_{1} \cup H$ is consistent iff $B_{2} \cup H$ is consistent. Hence, $B_{1}$ and $B_{2}$ are inductively equivalent under the minimal model semantics in Caulnd.

Theorem 3.6 (Condition for inductive equivalence under MM in learning from satisfiability) For any two clausal theories $B_{1}$ and $B_{2}, B_{1} \equiv{ }_{\mathrm{LFS}}^{M M} B_{2}$ iff $B_{1} \equiv B_{2}$.

Proof Suppose that $B_{1}$ and $B_{2}$ are inductively equivalent under the minimal model semantics in LFS. Then, for any set $H$ and for any set $O$ of clauses, $B_{1} \cup H \cup O$ is consistent iff $B_{2} \cup H \cup O$ is consistent. This condition reduces to that $B_{1} \cup H$ is consistent iff $B_{2} \cup H$ is consistent for any set $H$ of clauses $(*)$. If $B_{1} \not \equiv B_{2}$, there is a ground clause $C$ such that $B_{1} \models C$ but $B_{2} \not \models C$. Then, $B_{1} \cup\{\neg C\}$ is inconsistent, while $B_{2} \cup\{\neg C\}$ is consistent. As $\neg C$ is a conjunction of ground literals and is identified with a set of clauses, this contradicts the fact $(*)$. Thus, the condition implies the equivalence relation $B_{1} \equiv B_{2}$. The converse implication clearly holds.

Theorem 3.7 (Condition for inductive equivalence under MM in brave induction) For any two clausal theories $B_{1}$ and $B_{2}, B_{1} \equiv{ }_{\text {Bralnd }}^{M M} B_{2}$ iff $B_{1} \equiv B_{2}$.

Proof As inductive equivalence in Caulnd implies inductive equivalence in Bralnd (Proposition 3.1), $B_{1}$ and $B_{2}$ are inductively equivalent under the minimal model semantics in Bralnd if $B_{1} \equiv B_{2}$ by Theorem 3.5. On the other hand, inductive equivalence in Bralnd implies inductive equivalence in LFS (Proposition 3.1), so $B_{1}$ and $B_{2}$ are inductively equivalent under the minimal model semantics in Bralnd only if $B_{1} \equiv B_{2}$ by Theorem 3.6.

By the results of Theorems 3.5, 3.6 and 3.7, together with those results under the classical semantics, we conclude that

Theorem 3.8 (Identification of inductive equivalence in clausal theories) For any two clausal theories $B_{1}$ and $B_{2}, B_{1} \equiv{ }_{I}^{S E M} B_{2}$ iff $B_{1} \equiv B_{2}$ where SEM is either Mod or MM and $I \in\{$ Caulnd, Bralnd, LFS, DesInd $\}$.

Deciding the inductive equivalence of two theories is intractable in general. ${ }^{2}$

Proposition 3.9 (Complexity for deciding inductive equivalence between clausal theories) Deciding inductive equivalence of two propositional clausal theories is coNP-complete under both the classical semantics and the minimal model semantics in four different induction frameworks. The task is done in polynomial time when two theories are Horn.

Proof Given two propositional clausal theories $B_{1}$ and $B_{2}$, the problem of testing $B_{1} \equiv B_{2}$ is equivalent to the problem of testing unsatisfiability of $\left(B_{1} \wedge \neg B_{2}\right) \vee\left(\neg B_{1} \wedge B_{2}\right)$, which is coNP-complete. Then, the result follows by Theorem 3.8. Next, given two Horn logic programs $B_{1}$ and $B_{2}, B_{1} \supset B_{2}$ is checked by testing unsatisfiability of $B_{1} \cup\{\neg c\}$ for each clause $c \in B_{2}$. As Horn SAT is linear, this is done in quadratic time. The converse implication is checked in the same manner.

\footnotetext{
${ }^{2}$ Throughout the paper, complexity results are stated in terms of the size of input background theories.
} 


\section{Inductive equivalence in Horn ILP systems}

There are many ILP systems which use Horn logic programs as background theories. In these systems, the condition of inductive equivalence is often relaxed. In this section, we investigate inductive equivalence in some Horn ILP systems which are widely studied in the literature.

\subsection{FOIL}

FOIL (Quinlan 1990) induces function-free definite clauses which cover a positive observation and uncover a negative observation together with a background theory. Given a predicate $p$ to be learned, it starts with the fact $p\left(x_{1}, \ldots, x_{n}\right) \leftarrow$ which is then specialized using refinement operators that adds new literals to the body of the clause. FoIL repeatedly applies a refinement operator until the clause does not imply any fact included in a negative observation for the predicate. Once a clause is added to a hypothesis, every ground fact implied by that clause is deleted from a positive observation. The algorithm repeats the step until all facts in a positive observation are covered. FOIL uses an information-based heuristic to guide its search for hypotheses.

The logic for induction in FOIL is explanatory induction with a restricted problem setting. Given a function-free definite logic program $B$ (called a Datalog) and a set $O$ of ground facts, a hypothesis $H$ covers $O$ with respect to $B$ in FoIL if

$$
B \cup H \models O
$$

where $H$ is a set of function-free definite clauses satisfying the condition ${ }^{3}$ :

$$
H=\{C \mid \text { the predicate appearing in the head of a clause } C \text { appears in } O\} \text {. }
$$

The declarative semantics of a definite logic program $B$ is given by the unique minimal model $M_{B}$, called the least model. The least model has the model intersection property (Van Emden and Kowalski 1976) such that

$$
M_{B}=\bigcap_{M \in \operatorname{Mod}(B)} M
$$

Thus, the relation (3) is rewritten as

$$
E \subseteq M_{B \cup H}
$$

where $M_{B \cup H}$ is the least model of $B \cup H$. Note that $B \cup H$ is a definite logic program and is always consistent. By this fact, inductive equivalence in FOIL is defined as follows.

Definition 4.1 (Inductive equivalence in FOIL) Two Datalog programs $B_{1}$ and $B_{2}$ are inductively equivalent in FOIL if it holds that $O \subseteq M_{B_{1} \cup H}$ iff $O \subseteq M_{B_{2} \cup H}$ for any observation $O$ and for any hypothesis $H$.

\footnotetext{
${ }^{3}$ In Quinlan (1990), $H$ contains non-Horn clauses having negative literals in its body, but the author explains FOIL as a system for learning Horn clauses from data expressed as relations. To avoid ambiguity, here we assume $H$ as a set of Horn clauses which contain only atoms in their bodies.
} 
Let $H$ be a set of ground definite clauses and $M$ a set of ground atoms. Then, define

$$
T_{H}(M)=\left\{A \mid A \leftarrow A_{1}, \ldots, A_{n} \text { is in } H \text { and }\left\{A_{1}, \ldots, A_{n}\right\} \subseteq M\right\} .
$$

Theorem 4.1 (Condition for inductive equivalence in FOIL) Let $B_{1}$ and $B_{2}$ be two Datalog programs. Then, $B_{1}$ and $B_{2}$ are inductively equivalent in FOIL iff $B_{1} \equiv_{w} B_{2}$.

Proof For any $O$ and $H, O \subseteq M_{B_{1} \cup H}$ iff $O \subseteq M_{B_{2} \cup H}$

$$
\Leftrightarrow M_{B_{1} \cup H}=M_{B_{2} \cup H} \cdot(*)
$$

Putting $H=\emptyset$, (*) implies $M_{B_{1}}=M_{B_{2}}$. Hence, $B_{1} \equiv_{w} B_{2}$.

Conversely, if $B_{1} \equiv_{w} B_{2}$, then $M_{B_{1}}=M_{B_{2}}$. Suppose any set $H$ of ground clauses such that $H=\left\{A \leftarrow A_{1}, \ldots, A_{n} \mid A \in E\right\}$. Then, $M_{B_{1}} \cup T_{H}\left(M_{B_{1}}\right)=M_{B_{2}} \cup T_{H}\left(M_{B_{2}}\right)$. Since $M_{B_{i}} \cup T_{H}\left(M_{B_{i}}\right)=M_{B_{i} \cup H}$ for $i=1,2, M_{B_{1} \cup H}=M_{B_{2} \cup H}$. Hence, $O \subseteq M_{B_{1} \cup H}$ iff $O \subseteq M_{B_{2} \cup H}$ for any $O$ and $H$.

Example 4.1 Two programs

$$
\begin{aligned}
& B_{1}=\{p(x) \leftarrow q(x), r(a) \leftarrow\}, \\
& B_{2}=\{r(a) \leftarrow\}
\end{aligned}
$$

have the same least model $\{r(a)\}$, thereby weakly equivalent. Hence, $B_{1}$ and $B_{2}$ are inductively equivalent in FOIL.

In Example 4.1, $B_{1}$ and $B_{2}$ are not inductively equivalent in explanatory induction in general. In fact, for the observation $O=\{p(a)\}$, the hypothesis $H=\{q(x) \leftarrow r(x)\}$ explains $p(a)$ in $B_{1}$, but not in $B_{2}$. The hypothesis $H$ is not produced in FoIL, however, because the predicate $q$ appearing in the head does not appear in $O$.

\subsection{Golem}

Golem (Muggleton and Feng 1990) realizes explanatory induction in definite logic programs. It uses the algorithm of relative least generalization under subsumption (Plotkin 1971). We first review basic terms and results. A clause $C_{1}$ subsumes another clause $C_{2}$ relative to a program $B$, denoted by $C_{1} \succeq_{B} C_{2}$, if there is a substitution $\theta$ such that $B \models C_{1} \theta \rightarrow C_{2}$. A clause $D$ is a relative least generalization under subsumption (rlgs) of $C_{1}$ and $C_{2}$ with respect to $B$ if $D$ is the least upper bound of $C_{1}$ and $C_{2}$ under the ordering $\succeq_{B}$ over the clausal language. The $r l g s$ does not always exist but exists when $B$ is a set of ground atoms (Nienhuys-Cheng and De Wolf 1997).

Given a definite logic program $B$ and a set $O$ of ground facts, GoLEM constructs a hypothesis $H$ as follows:

$$
\begin{aligned}
& B \cup H \models O \\
& \quad \Leftrightarrow \quad H \models B \rightarrow O \\
& \quad \Leftrightarrow \quad \models H \rightarrow(\neg B \vee O) .
\end{aligned}
$$

At this point, GoLEM replaces $B$ with the conjunction of ground atoms included in a finite subset of the least model $M_{B}$ of $B$. For simplicity reasons, we suppose that the least model $M_{B}$ is finite and replace $B$ with $M_{B}$. Let $O=\left\{A_{1}, \ldots, A_{k}\right\}$. Then,

$$
H \rightarrow \neg M_{B} \vee O
$$


where

$$
\neg M_{B} \vee O=\left(A_{1} \vee \neg M_{B}\right) \wedge \cdots \wedge\left(A_{k} \vee \neg M_{B}\right)
$$

with $\neg M_{B}=\bigvee_{A_{i} \in M_{B}} \neg A_{i}$. Next, the $\operatorname{rlgs}$ of $O$ with respect to $M_{B}$ (written as $\operatorname{rlgs}\left(M_{B}, O\right)$ ) is computed as the least generalization under subsumption (lgs) of clauses $\left(A_{1} \vee\right.$ $\left.\neg M_{B}\right), \ldots,\left(A_{k} \vee \neg M_{B}\right)$ (written as $\lg s\left(A_{1} \vee \neg M_{B}, \ldots, A_{k} \vee \neg M_{B}\right)$ ). A hypothesis $H$ is then put as

$$
H=\operatorname{rgs}\left(M_{B}, O\right)
$$

which is a set of definite clauses.

Inductive equivalence in GOLEM is now defined as follows.

Definition 4.2 (Inductive equivalence in GOLEM) Let $B_{1}$ and $B_{2}$ be two definite logic programs such that each program has the least model as a finite set. Then, $B_{1}$ and $B_{2}$ are inductively equivalent in GOLEM if $\operatorname{rgs}\left(M_{B_{1}}, O\right)=\operatorname{rlg} s\left(M_{B_{2}}, O\right)$ for any set $O$ of ground facts.

We then have the following result.

Theorem 4.2 (Condition for inductive equivalence in Golem) Let $B_{1}$ and $B_{2}$ be two definite logic programs. Then, $B_{1}$ and $B_{2}$ are inductively equivalent in GOLEM iff $B_{1} \equiv_{w} B_{2}$.

Proof Suppose that $B_{1}$ and $B_{2}$ are inductively equivalent in GoLEM. Then, for any set $O=\left\{A_{1}, \ldots, A_{k}\right\}$ of ground facts, $\operatorname{rlgs}\left(M_{B_{1}}, O\right)=\operatorname{rlgs}\left(M_{B_{2}}, O\right)$ implies $\operatorname{lgs}\left(A_{1} \vee\right.$ $\left.\neg M_{B_{1}}, \ldots, A_{k} \vee \neg M_{B_{1}}\right)=\lg s\left(A_{1} \vee \neg M_{B_{2}}, \ldots, A_{k} \vee \neg M_{B_{2}}\right)$. Put $O=\{A\}$ for any ground atom $A$. Then, $\lg s\left(A \vee \neg M_{B_{1}}\right)=\lg s\left(A \vee \neg M_{B_{2}}\right)$ implies $A \vee \neg M_{B_{1}}=A \vee \neg M_{B_{2}}$ thereby $M_{B_{1}}=M_{B_{2}}$. Hence, $B_{1} \equiv_{w} B_{2}$.

Conversely, if $B_{1} \equiv_{w} B_{2}, M_{B_{1}}=M_{B_{2}}$. Then, for any set $O=\left\{A_{1}, \ldots, A_{k}\right\}$ of ground facts, $\lg s\left(A_{1} \vee \neg M_{B_{1}}, \ldots, A_{k} \vee \neg M_{B_{1}}\right)=\lg s\left(A_{1} \vee \neg M_{B_{2}}, \ldots, A_{k} \vee \neg M_{B_{2}}\right)$, so $\operatorname{rlgs}\left(M_{B_{1}}, O\right)=\operatorname{rlgs}\left(M_{B_{2}}, O\right)$. Hence, the result holds.

Example 4.2 Consider two programs:

$$
\begin{aligned}
B_{1}= & \{\text { has_wings }(\text { joe }) \leftarrow \operatorname{bird}(\text { joe }), \\
& \text { bird }(\text { tweety }) \leftarrow, \\
& \text { bird }(\text { polly }) \leftarrow\}, \\
B_{2}= & \{\text { bird }(\text { tweety }) \leftarrow, \\
& \operatorname{bird}(\text { polly }) \leftarrow\} .
\end{aligned}
$$

Given the observation $O=\{$ flies(tweety), flies(polly) $\}$, both $r \lg s\left(M_{B_{1}}, O\right)$ and $r \lg s\left(M_{B_{2}}, O\right)$ contain the single clause:

$$
\operatorname{flies}(x) \leftarrow \operatorname{bird}(x) \text {. }
$$

This means that the first clause of $B_{1}$ is of no use for induction in GoLEM. Note that $B_{1}$ and $B_{2}$ are weakly equivalent, but they are not logically equivalent. 
In the process of constructing inductive hypothesis $H$, GOLEM approximates $B$ to a finite subset of $M_{B}$. However, $\operatorname{rlgs}(B, O) \neq \operatorname{rlgs}\left(M_{B}, O\right)$ in general. In fact, in Example 4.2, given $O=\{$ has_wing (joe $)\}$, the hypothesis $H=\{$ bird(joe $)\}$ is obtained in $B_{1}$ but not in $B_{2}$. This means that some hypotheses which are computed under $r l g s$ might be lost by GOLEM.

\subsection{PROGOL}

PROGOL is also known as a Horn ILP system which realizes explanatory induction. It is based on the inverse entailment algorithm developed in Muggleton (1995). Given a Horn logic program $B$ and a ground Horn clause $O$ as an observation, suppose a Horn clause $H$ satisfying

$$
B \cup\{H\} \models O .
$$

By inverting the entailment relation it becomes

$$
B \cup\{\neg O\} \models \neg H .
$$

Put $\neg \operatorname{bot}(B, O)$ as the conjunction of ground literals which are true in every model of $B \cup$ $\{\neg O\}$. Then, a clause $H$ is induced by inverse entailment (IE) if $H \models \operatorname{bot}(B, E)$ where $\operatorname{bot}(B, E)$ is a clause called a bottom clause. ${ }^{4}$

Inductive equivalence in PROGOL is defined as follows.

Definition 4.3 (Inductive equivalence in Progol) Two Horn logic programs $B_{1}$ and $B_{2}$ are inductively equivalent in PROGOL if $\operatorname{bot}\left(B_{1}, O\right)=\operatorname{bot}\left(B_{2}, O\right)$ for any ground Horn clause $O$.

Then, we have the following result.

Theorem 4.3 (Condition for inductive equivalence in PROGOL) Two Horn logic programs $B_{1}$ and $B_{2}$ are inductively equivalent under PROGOL iff $B_{1} \equiv B_{2}$.

Proof $B_{1}$ and $B_{2}$ are inductively equivalent under PROGOL iff $\operatorname{bot}\left(B_{1}, O\right)=\operatorname{bot}\left(B_{2}, O\right)$ for any $O$. Then, $\neg \operatorname{bot}\left(B_{1}, O\right)=\neg \operatorname{bot}\left(B_{2}, O\right)$, and $B_{1} \cup\{\neg O\} \models L$ iff $B_{2} \cup\{\neg O\} \models L$ for any ground Horn clause $O$ and for any ground literal $L$. Put $O=\leftarrow A_{1}, \ldots, A_{n}$. Then, $B_{1} \cup\left\{A_{1}, \ldots, A_{n}\right\} \models L$ iff $B_{2} \cup\left\{A_{1}, \ldots, A_{n}\right\} \models L$ for any $\left\{A_{1}, \ldots, A_{n}\right\}$. Thus, for any finite set $F$ of ground atoms, $B_{1} \cup F \models L$ iff $B_{2} \cup F \models L$. So, $B_{1} \models F \supset L$ iff $B_{2} \models F \supset L$ for any finite set $F$ of ground atoms and any ground literal $L$. This implies $B_{1} \equiv B_{2}$. Conversely, $B_{1} \equiv B_{2}$ implies $\operatorname{bot}\left(B_{1}, O\right)=\operatorname{bot}\left(B_{2}, O\right)$, hence the result holds.

In PROGOL, weak equivalence of two programs is not sufficient for inductive equivalence.

\footnotetext{
${ }^{4}$ Strictly speaking, Progol does not produce every clause satisfying the relation $H \models \operatorname{bot}(B, E)$ and is in this sense incomplete (Badea and Stanciu 1999). But here we proceed our discussion by assuming an ideal algorithm which computes every $H$ satisfying the relation. $C F$-induction (Inoue 2004) realizes a sound and complete induction algorithm based on IE in full clausal theories.
} 
Example 4.3 Consider two programs:

$$
\begin{aligned}
B_{1}= & \{\text { white_swan }(c) \leftarrow\} \\
B_{2}= & \{\text { abnormal }(x) \leftarrow \text { black_swan }(x), \\
& \text { white_swan }(c) \leftarrow\} .
\end{aligned}
$$

Given the observation $O=\leftarrow$ black_swan $(c)$, it becomes

$$
\neg \text { bot }\left(B_{1}, O\right)=\text { white_swan }(c) \wedge \text { black_swan }(c) .
$$

Then,

$$
H_{1}=\leftarrow \text { white_swan }(x), \text { black_swan }(x)
$$

becomes a hypothesis satisfying $H_{1} \models \operatorname{bot}\left(B_{1}, E\right)$. By contrast,

$$
\neg \text { bot }\left(B_{2}, O\right)=\text { white_swan }(c) \wedge \text { black_swan }(c) \wedge \text { abnormal }(c) .
$$

Then,

$$
H_{2}=\leftarrow \operatorname{abnormal}(x)
$$

becomes a hypothesis satisfying $H_{2} \models \operatorname{bot}\left(B_{2}, E\right)$. Note that $B_{1} \not \equiv B_{2}$ but $B_{1} \equiv_{w} B_{2}$.

\subsection{Claudien}

The system Claudien (De Raedt and Bruynooghe 1993; De Raedt and Dehaspe 1997b) realizes descriptive induction under the completion semantics (Clark 1978). Given a definite logic program $B$ and a set $O$ of definite clauses, CLAUDIEN produces a set $H$ of clauses satisfying

$$
\operatorname{Comp}(B \cup O) \models H
$$

where Comp represents Clark's predicate completion.

Example 4.4 Let $B=\{\operatorname{human}(s)\}$ and $O=\{\operatorname{mortal}(s)\}$. Then, the following clauses are all possible solutions:

$$
\begin{aligned}
& H_{1}=\{\operatorname{mortal}(x) \leftarrow \operatorname{human}(x)\}, \\
& H_{2}=\{\operatorname{human}(x) \leftarrow \operatorname{mortal}(x)\}, \\
& H_{3}=\{\operatorname{human}(x) \vee \operatorname{mortal}(x) \leftarrow\} .
\end{aligned}
$$

Note that in descriptive induction it is assumed that the universe defined by an observation together with a background theory is completely specified (De Raedt and Lavrač 1993).

Definition 4.4 (Inductive equivalence in CLAUDIEN) Let $B_{1}$ and $B_{2}$ be two definite logic programs. Then, $B_{1}$ and $B_{2}$ are inductively equivalent in CLAUDIEN if it holds that

$$
\operatorname{Comp}\left(B_{1} \cup O\right) \models H \quad \text { iff } \quad \operatorname{Comp}\left(B_{2} \cup O\right) \models H
$$

for any observation $O$ and for any hypothesis $H$ such that $B_{1} \cup O$ and $B_{2} \cup O$ are consistent. 
Table 1 Comparison of Horn ILP systems

\begin{tabular}{llll}
\hline System & Language & Induction & Condition \\
\hline FOIL & Datalog & Explnd & $B_{1} \equiv w B_{2}$ \\
GOLEM & Definite LP & Explnd & $B_{1} \equiv w B_{2}$ \\
PROGOL & Horn LP & Explnd & $B_{1} \equiv B_{2}$ \\
CLAUDIEN & Definite LP & DesInd & $B_{1} \equiv B_{2}$ \\
\hline
\end{tabular}

Theorem 4.4 (Condition for inductive equivalence in CLAUDIEN) Two definite logic programs $B_{1}$ and $B_{2}$ are inductively equivalent in CLAUDIEN iff $B_{1} \equiv B_{2}$.

Proof It is shown that $B_{1} \equiv B_{2}$ iff $\operatorname{Comp}\left(B_{1} \cup O\right) \equiv \operatorname{Comp}\left(B_{2} \cup O\right)$ for any clausal theory $O$. The proof is similar to Proposition 2.2.

The results of Sect. 4 are summarized in Table 1. Observe that the conditions of inductive equivalence in FOIL and GOLEM are weaker than the condition of inductive equivalence in explanatory induction (Theorem 3.2). This is due to the fact that these systems impose some restrictions on the syntax of background theories, observations and hypotheses. By contrast, the condition of inductive equivalence in PROGOL and CLAUDIEN is identical to the one in clausal logic.

\section{Inductive equivalence in nonmonotonic logic programs}

\subsection{Nonmonotonic logic programs}

Nonmonotonic logic programs are logic programs with negation as failure (Baral and Gelfond 1994). We consider the class of extended disjunctive programs (Gelfond and Lifschitz 1991 ) in this paper. An extended disjunctive program (EDP) (or simply a program) is a set of rules of the form:

$$
L_{1} ; \cdots ; L_{l} \leftarrow L_{l+1} \ldots, L_{m}, \text { not } L_{m+1}, \ldots, \text { not } L_{n} \quad(n \geq m \geq l \geq 0)
$$

where each $L_{i}$ is a positive/negative literal, i.e., $A$ or $\neg A$ for an atom $A$, and not is negation as failure (NAF). not $L$ is called an NAF-literal. The symbol ";" represents disjunction. The left-hand side of " $\leftarrow$ " is the head, and the right-hand side is the body. For each rule $r$ of the form (4), head(r), $\operatorname{body}^{+}(r)$ and $\operatorname{body}^{-}(r)$ denote the sets of literals $\left\{L_{1}, \ldots, L_{l}\right\}$, $\left\{L_{l+1}, \ldots, L_{m}\right\}$, and $\left\{L_{m+1}, \ldots, L_{n}\right\}$, respectively. Also, not_body ${ }^{-}(r)$ denotes the set of NAF-literals $\left\{\right.$ not $L_{m+1}, \ldots$, not $\left.L_{n}\right\}$. A disjunction of literals and a conjunction of (NAF-) literals in a rule are identified with its corresponding sets of literals. A rule $r$ is disjunctive if head(r) contains more than one literal. A rule $r$ is a constraint if head $(r)=\emptyset$; and $r$ is a fact if $\operatorname{bod} y(r)=\emptyset$. A program is basic if no rule contains NAF-literals. A program, rule, or literal is ground if it contains no variable. A propositional program is a finite set of ground rules. A program $P$ with variables is a shorthand of its ground instantiation ground $(P)$, the (possibly infinite) set of ground rules obtained from $P$ by substituting variables in $P$ by elements of its Herbrand universe in every possible way. A program is called an extended logic program (ELP) if it contains no disjunctive rule. An ELP is called a normal logic program (NLP) if every literal $L_{i}$ appearing in the program is an atom. 
A primary difference between nonmonotonic logic programs and clausal theories is that a rule (4) is not a clause even if it contains no NAF-literal. For instance, a rule $L_{1} \leftarrow L_{2}$ has meaning different from $\neg L_{2} \leftarrow \neg L_{1}$ or $L_{1} \vee \neg L_{2}$. The rule (4) is interpreted as an inference rule rather than an implication formula (Gelfond and Lifschitz 1991). Thus, induction in nonmonotonic logic programs is different from induction in clausal theories.

The semantics of an EDP is defined by the answer set semantics (Gelfond and Lifschitz 1991). The literal base Lit is the set of all ground literals in the language of a program. Suppose a program $P$ and a set of literals $S\left(\subseteq\right.$ Lit). Then, the reduct $P^{S}$ is the program which contains the ground rule $h e a d(r) \leftarrow \operatorname{body}^{+}(r)$ iff there is a rule $r$ in $\operatorname{ground}(P)$ such that $\operatorname{body}^{-}(r) \cap S=\emptyset$. Given a basic program $P$, let $S$ be a set of ground literals that is (i) closed under $P$, i.e., for every ground rule $r$ in $\operatorname{ground}(P), \operatorname{bod} y(r) \subseteq S$ implies head $(r) \cap$ $S \neq \emptyset$; and (ii) logically closed, i.e., it is either consistent or equal to Lit. An answer set of a basic program $P$ is a minimal set $S$ satisfying both (i) and (ii). Given an EDP $P$ and a set $S$ of ground literals, $S$ is an answer set of $P$ if $S$ is an answer set of $P^{S}$. A program has none, one, or multiple answer sets in general. The set of all answer sets of $P$ is written as $A S(P)$. Here $A S(P)$ is an antichain set, i.e., no element $S \in A S(P)$ is a proper subset of another element $T \in A S(P)$. An answer set is consistent if it is not Lit. A program $P$ is consistent if it has a consistent answer set; otherwise, $P$ is inconsistent. In normal logic programs, answer sets are also called stable models (Gelfond and Lifschitz 1988). A set $S$ of ground literals satisfies a ground rule $r$ if either $S \cap \operatorname{head}(r) \neq \emptyset$, $\operatorname{body}^{+}(r) \backslash S \neq \emptyset$ or $\operatorname{body}^{-}(r) \cap S \neq \emptyset$. When a rule $r$ contains variables, $S$ satisfies $r$ if $S$ satisfies every ground instance of $r$. $S$ satisfies a set $R$ of rules if $S$ satisfies every rule in $R$. A program $P$ satisfies a set $R$ of rules if every answer set of $P$ satisfies every rule in $R$. A program $P$ is consistent if it has an answer set; otherwise $P$ is inconsistent.

Example 5.1 Let $P$ be the program:

$$
\begin{aligned}
& p(x) \leftarrow \operatorname{not} q(x), \\
& q(x) \leftarrow \operatorname{not} p(x), \\
& r(a) \leftarrow
\end{aligned}
$$

where $A S(P)=\{\{p(a), r(a)\},\{q(a), r(a)\}\}$. Then, every answer set satisfies the rule $p(a) ; q(a) \leftarrow$, while $p(a) \leftarrow r(a)$ is satisfied by $\{p(a), r(a)\}$, but not by $\{q(a), r(a)\}$.

\subsection{Inductive equivalence between EDPs}

Four different induction frameworks in Sect. 2.2 are applied to induction in nonmonotonic logic programs. In this section, we consider the following problem setting:

- a background theory $B$ is given as an EDP under the answer set semantics $\operatorname{SEM}(B)=$ $A S(B)$

- an observation $O$ is a set of rules

- a hypothesis $H$ is a set of rules

Note that in case of nonmonotonic logic programs, a background theory $B$ could be inconsistent. In this case, the introduction of $H$ to $B$ makes $B \cup H$ consistent. This is the difference from the case of monotonic background theories. In case of monotonic theories, an inconsistent $B$ cannot become consistent by introducing any $H$. 
Example 5.2 Let $B$ be the program:

$$
p \leftarrow \text { not } p
$$

Then, $B$ is inconsistent, i.e., $A S(B)=\emptyset$. Putting $H=\{p \leftarrow\}, B \cup H$ becomes consistent, i.e., $A S(B \cup H)=\{\{p\}\}$.

Four different definitions of inductive equivalence are then considered under the answer set semantics.

Definition 5.1 (Inductive equivalence in different frameworks of induction) Let $B_{1}$ and $B_{2}$ be two programs having the same literal base Lit. Then,

1. $B_{1}$ and $B_{2}$ are inductively equivalent under the answer set semantics in cautious induction (written $B_{1} \equiv{ }_{\text {Caulnd }}^{A S} B_{2}$ ) if for any $O$ and any $H, O$ is satisfied by every $S \in A S\left(B_{1} \cup H\right)$ iff $O$ is satisfied by every $S \in A S\left(B_{2} \cup H\right)$, where $B_{1} \cup H$ and $B_{2} \cup H$ are consistent.

2. $B_{1}$ and $B_{2}$ are inductively equivalent under the answer set semantics in brave induction (written $B_{1} \equiv{ }_{\text {Bralnd }}^{A S} B_{2}$ ) if for any $O$ and any $H, O$ is satisfied by some $S \in A S\left(B_{1} \cup H\right)$ iff $O$ is satisfied by some $S \in A S\left(B_{2} \cup H\right)$, where $B_{1} \cup H$ and $B_{2} \cup H$ are consistent.

3. $B_{1}$ and $B_{2}$ are inductively equivalent under the answer set semantics in learning from satisfiability (written $B_{1} \equiv{ }_{\mathrm{LFS}}^{A S} B_{2}$ ) if for any $O$ and any $H, B_{1} \cup H \cup O$ is consistent iff $B_{2} \cup H \cup O$ is consistent.

4. $B_{1}$ and $B_{2}$ are inductively equivalent under the answer set semantics in descriptive induction (written $B_{1} \equiv{ }_{\text {Desind }}^{A S} B_{2}$ ) if for any $O$ and any $H, H$ is satisfied by every $S \in A S\left(B_{1} \cup O\right)$ iff $H$ is satisfied by every $S \in A S\left(B_{2} \cup O\right)$, where $B_{1} \cup O$ and $B_{2} \cup O$ are consistent.

In each case, we say that a hypothesis $H$ covers (or explains) $O$ with respect to $B$ under the answer set semantics in the induction framework $I$. Here, $I$ is one of the four induction frameworks presented above.

Next we provide a program transformation which is useful for subsequent discussion. Given a set $O$ of ground rules, any rule $r$ in $O$ is transformed to the set $\Omega$ of rules:

$$
\begin{array}{ll}
G_{r} \leftarrow L_{i} & \text { for every } L_{i} \in \operatorname{head}(r), \\
G_{r} \leftarrow \operatorname{not} L_{j} & \text { for every } L_{j} \in \operatorname{body}(r), \\
G_{r} \leftarrow L_{k} & \text { for every } L_{k} \in \operatorname{body}^{-}(r),
\end{array}
$$

where $G_{r}$ is a new ground atom appearing nowhere in $B$ and uniquely associated with each $r$. With this setting, the next result holds.

Proposition 5.1 $O$ is satisfied by an answer set of $B \cup H$ iff for any $r \in O, G_{r}$ is included in an answer set of $B \cup H \cup \Omega$.

Proof $O$ is satisfied by an answer set $S$ of $B \cup H$

iff for any $r$ in $O$, either $S \cap \operatorname{head}(r) \neq \emptyset, \operatorname{body}^{+}(r) \backslash S \neq \emptyset$ or $\operatorname{body}^{-}(r) \cap S \neq \emptyset$ for some $S \in A S(B \cup H)$

iff $B \cup H \cup \Omega$ has an answer set $T=S \cup\left\{G_{r} \mid r \in O\right\}$ for some $S \in A S(B \cup H)$.

Thus, any observation $O$ as a set of rules is instantiated to its ground instances $\operatorname{ground}(O)$, which is then transformed to a semantically equivalent observation as a set of ground atoms. 
Proposition 5.2 (Relations between different inductive equivalences) The following relations hold between equivalence relations in different induction.

1. $B_{1} \equiv \underset{\text { Caulnd }}{A S} B_{2}$ implies $B_{1} \equiv \underset{\text { Bralnd }}{A S} B_{2}$.

2. $B_{1} \equiv{ }_{\text {Bralnd }}^{A S} B_{2}$ iff $B_{1} \equiv{ }_{\mathrm{LFS}}^{A S} B_{2}$.

3. $B_{1} \equiv{ }_{\text {Caulnd }}^{A S} B_{2}$ iff $B_{1} \equiv \underset{\text { Desind }}{A S} B_{2}$.

Proof The results (1) and (3) follow from definitions. We show (2). If $B_{1} \equiv{ }_{\mathrm{LFS}}^{A S} B_{2}$, for any $H$ and any $O, B_{1} \cup H \cup \Omega \cup\left\{\leftarrow\right.$ not $\left.G_{r} \mid r \in \operatorname{ground}(O)\right\}$ is consistent iff $B_{2} \cup H \cup \Omega \cup\{\leftarrow$ not $\left.G_{r} \mid r \in \operatorname{ground}(O)\right\}$ is consistent. Put $U=\left\{G_{r} \mid r \in \operatorname{ground}(O)\right\}$. Then, for any $H$ and any $U, U \subseteq S$ for some consistent answer set $S$ of $B_{1} \cup H \cup \Omega$ iff $U \subseteq T$ for some consistent answer set $T$ of $B_{2} \cup H \cup \Omega$. Hence, $B_{1} \equiv \underset{\text { Bralnd }}{A S} B_{2}$. The only-if part clearly holds.

Proposition 5.2(2) presents that the notions of inductive equivalence in brave induction and learning from satisfiability coincide under the answer set semantics.

We proceed to build conditions for inductive equivalence between EDPs.

Theorem 5.3 (Condition for inductive equivalence under AS in cautious induction) Let $B_{1}$ and $B_{2}$ be any EDPs. Then, $B_{1} \equiv_{s} B_{2}$ implies $B_{1} \equiv{ }_{\text {Caulnd }}^{A S} B_{2}$. The converse implication also holds for any $H$ such that $A S\left(B_{1} \cup H\right)$ and $A S\left(B_{2} \cup H\right)$ are finite sets. ${ }^{5}$

Proof If $B_{1} \equiv_{s} B_{2}, A S\left(B_{1} \cup H\right)=A S\left(B_{2} \cup H\right)$ holds for any set $H$ of rules. In this case, $O$ is satisfied by every answer set of $B_{1} \cup H$ iff $O$ is satisfied by every answer set of $B_{2} \cup H$ for any $O$ and $H$ such that $B_{1} \cup H$ and $B_{2} \cup H$ are consistent. Hence, $B_{1}$ and $B_{2}$ are inductively equivalent in cautious induction.

Conversely, suppose that $B_{1}$ and $B_{2}$ are inductively equivalent in cautious induction. Then, it holds that $O$ is satisfied in every answer set of $B_{1} \cup H$ iff $O$ is satisfied in every answer set of $B_{2} \cup H$ for any $O$ and any $H$ such that $B_{1} \cup H$ and $B_{2} \cup H$ are consistent. Suppose that there is a set $S$ such that $S \in A S\left(B_{1} \cup H\right) \backslash A S\left(B_{2} \cup H\right)$ for some $H$. For any answer set $T_{i}$ of $B_{2} \cup H$, put

$$
\bigcup_{i}\left(S \backslash T_{i}\right)=U \text { and } \bigcup_{i}\left(T_{i} \backslash S\right)=V .
$$

For some non-empty finite subset of $U^{\prime} \subseteq U$ and $V^{\prime} \subseteq V$, construct the constraint

$$
C: \leftarrow U^{\prime}, \text { not } V^{\prime}
$$

where $U^{\prime}$ or $V^{\prime}$ is identified with the conjunction of literals included in each set. By $U^{\prime} \subseteq S$ and $V^{\prime} \cap S=\emptyset, S$ does not satisfy $C$.

If every answer set $T_{i}$ of $B_{2} \cup H$ satisfies $C$, this contradicts the assumption that $B_{1}$ and $B_{2}$ are inductively equivalent. Else if some answer set $T_{i}$ of $B_{2} \cup H$ does not satisfy $C, U^{\prime} \subseteq T_{i}$ and $V^{\prime} \cap T_{i}=\emptyset$. For every such $T_{i}$, either $S \backslash T_{i} \neq \emptyset$ or $T_{i} \backslash S \neq \emptyset$ holds by $S \notin A S\left(B_{2} \cup H\right)$. For every $T_{i}$ satisfying $S \backslash T_{i} \neq \emptyset$, take one literal $L_{i}$ from $S \backslash T_{i}$ and collect such a literal from each $T_{i}$. Put the collection as $W_{1}$ :

$$
W_{1}=\bigcup_{i}\left\{L_{i} \mid L_{i} \in S \backslash T_{i} \text { where } S \backslash T_{i} \neq \emptyset\right\} .
$$

\footnotetext{
${ }^{5}$ At the moment, the result is open when a program has an infinite number of answer sets.
} 
Similarly, for every $T_{i}$ satisfying $T_{i} \backslash S \neq \emptyset$, take one literal $L_{i}$ from $T_{i} \backslash S$ and collect such a literal from each $T_{i}$. Put the collection as $W_{2}$ :

$$
W_{2}=\bigcup_{i}\left\{L_{i} \mid L_{i} \in T_{i} \backslash S \text { where } T_{i} \backslash S \neq \emptyset\right\} .
$$

Here, $W_{1}$ and $W_{2}$ are finite set, because $B_{2} \cup H$ has a finite number of answer sets. Suppose the constraint

$$
D: \leftarrow U, W_{1} \text {, not } V \text {, not } W_{2} .
$$

As $W_{1} \nsubseteq T_{i}$ or $W_{2} \cap T_{i} \neq \emptyset$ holds for any $T_{i}, T_{i}$ satisfies $D$. Thus, $D$ is satisfied by every answer set of $B_{2} \cup H$. On the other hand, $W_{1} \subseteq S$ and $W_{2} \cap S=\emptyset$ imply that $S$ does not satisfy $D$. Then, $D$ is not satisfied by some answer set of $B_{1} \cup H$. This contradicts the assumption that $B_{1}$ and $B_{2}$ are inductively equivalent.

Theorem 5.4 (Condition for inductive equivalence under AS in brave induction) Let $B_{1}$ and $B_{2}$ be any EDPs. Then, $B_{1} \equiv \underset{\text { Bralnd }}{A S} B_{2}$ iff $B_{1} \equiv_{s} B_{2}$.

Proof When $B_{1}$ and $B_{2}$ are inductively equivalent in brave induction, it holds that $O$ is satisfied in an answer set of $B_{1} \cup H$ iff $O$ is satisfied in an answer set of $B_{2} \cup H$ for any $O$ and any $H$ such that $B_{1} \cup H$ and $B_{2} \cup H$ are consistent. Then, for any set $O$ of ground literals, $O \subseteq S$ for an answer set $S$ of $B_{1} \cup H$ iff $O \subseteq T$ for an answer set $T$ of $B_{2} \cup H$. Putting $O=S, S$ is an answer set of $B_{1} \cup H$ iff $S \subseteq T$ for an answer set $T$ of $B_{2} \cup H$ (*). Putting $O=T, T$ is an answer set of $B_{2} \cup H$ iff $T \subseteq S^{\prime}$ for an answer set $S^{\prime}$ of $B_{1} \cup H(\dagger)$. By (*) and ( $\dagger), S$ is an answer set of $B_{1} \cup H$ iff $S \subseteq T \subseteq S^{\prime}$ for an answer set $T$ of $B_{2} \cup H$ and for an answer set $S^{\prime}$ of $B_{1} \cup H$. Since $A S\left(B_{1} \cup H\right)$ is an antichain set, $S=T$. Thus, $S$ is an answer set of $B_{1} \cup H$ iff $S$ is an answer set of $B_{2} \cup H$ for any $H$. Hence, $B_{1}$ and $B_{2}$ are strongly equivalent.

Conversely, if $B_{1} \equiv_{s} B_{2}, A S\left(B_{1} \cup H\right)=A S\left(B_{2} \cup H\right)$ for any set $H$ of rules. Then, $O$ is satisfied in an answer set of $B_{1} \cup H$ iff $O$ is satisfied in an answer set of $B_{2} \cup H$ for any set $O$ of rules. Hence, $B_{1} \equiv \underset{\text { Bralnd }}{A S} B_{2}$ holds.

Theorem 5.5 (Condition for inductive equivalence under AS in learning from satisfiability) Let $B_{1}$ and $B_{2}$ be any EDPs. Then, $B_{1} \equiv{ }_{\mathrm{LFS}}^{A S} B_{2}$ iff $B_{1} \equiv_{s} B_{2}$.

Proof The result holds by Theorems 5.2(2) and 5.4.

The complexity of testing strong equivalence of two propositional EDPs is coNPcomplete (Turner 2003). Hence we have the next result.

Proposition 5.6 (Complexity for deciding inductive equivalence between EDPs) Deciding inductive equivalence of two propositional EDPs is coNP-complete under the answer set semantics in four different induction frameworks.

\subsection{Inductive equivalence in nonmonotonic ILP systems}

In this section, we investigate inductive equivalence in two nonmonotonic ILP systems. 


\subsubsection{Induction of stable models}

Otero (2001) characterizes induction problems in normal logic programs (NLPs) under the stable model semantics. Recall that an NLP is a set of rules of the form:

$$
A_{0} \leftarrow A_{1}, \ldots, A_{m}, \text { not } A_{m+1}, \ldots, \text { not } A_{n} \quad(n \geq m \geq 0)
$$

where each $A_{i}$ is an atom. Answer sets coincide with stable models in NLPs, so that Otero's framework is considered a special case of induction under the answer set semantics. Otero introduces different types of induction for positive/negative observations, but here we consider the so-called induction from non-complete sets which is the usual ILP setting for positive observations.

Suppose a background theory $B$ as an NLP, and a set $O$ of ground atoms as a positive observation such that $O$ is not satisfied by $B$. The goal is to find a set $H$ of rules satisfying the condition that $O$ is satisfied by every stable model of $B \cup H$. Thus, Otero's framework realizes cautious induction in nonmonotonic logic programs. An interpretation $M$ is a monotonic model of an NLP if $M$ satisfies every rule in $B$. A stable model is a monotonic model, but not vice versa. Given an observation $O$, an interpretation $M$ is an extension of $O$ iff $O \subseteq M$. He then captures the computation of $H$ as an extension $M$ of $O$ that becomes a stable model of $B \cup M$. That is, $H=M$ satisfying $O \subseteq M$ and $M \in A S(B \cup M)$ becomes a solution. Note that in this definition a hypothesis $H$ is given as a set of ground atoms.

Let $\operatorname{ISM}(B, O)$ be the collection of $H$ defined as above. Then, inductive equivalence in induction of stable models (ISM) is defined as follows.

Definition 5.2 (Inductive equivalence in ISM) Two NLPs $B_{1}$ and $B_{2}$ are inductively equivalent in $I S M$ if $\operatorname{ISM}\left(B_{1}, O\right)=\operatorname{ISM}\left(B_{2}, O\right)$ for any set $O$ of ground atoms.

Proposition 5.7 (Otero 2001) Given an NLP B, M is a monotonic model of B iff $M$ is a stable model of $B \cup M$.

Let $\operatorname{Mon} \operatorname{Mod}(B)$ be the set of monotonic models of $B$. Then we have the following result.

Theorem 5.8 (Condition for inductive equivalence in ISM) Two NLPs $B_{1}$ and $B_{2}$ are inductively equivalent in ISM iff $\operatorname{MonMod}\left(B_{1}\right)=\operatorname{MonMod}\left(B_{2}\right)$.

Proof Suppose that $B_{1}$ and $B_{2}$ are inductively equivalent in $\operatorname{ISM}$. For any $M \in \operatorname{ISM}\left(B_{1}, O\right)$, $M$ is a stable model of $B_{1} \cup M$ and a monotonic model of $B_{1}$ (Proposition 5.7). Then, $\operatorname{ISM}\left(B_{1}, O\right)=\operatorname{ISM}\left(B_{2}, O\right)$ implies $\operatorname{MonMod}\left(B_{1}\right)=\operatorname{MonMod}\left(B_{2}\right)$. Conversely, if $\operatorname{MonMod}\left(B_{1}\right)=\operatorname{MonMod}\left(B_{2}\right)$, for any set $M$ of atoms, $M$ is a stable model of $B_{1} \cup M$ iff $M$ is a stable model of $B_{2} \cup M$. Then, for any set $O$ of ground atoms, $M(\supseteq O)$ is a stable model of $B_{1} \cup M$ iff $M$ is a stable model of $B_{2} \cup M$. Hence, $\operatorname{ISM}\left(B_{1}, O\right)=\operatorname{ISM}\left(B_{2}, O\right)$.

Example 5.3 Let $B_{1}=\{p \leftarrow$ not $q\}$ and $B_{2}=\{q \leftarrow$ not $p\}$. For $O=\{p\}$, put its extension as $M=\{p\}$. Then, $H=\{p \leftarrow\}$ becomes a solution in both $B_{1}$ and $B_{2}$. Note that $B_{1} \not \equiv_{w} B_{2}$ but $\operatorname{MonMod}\left(B_{1}\right)=\operatorname{MonMod}\left(B_{2}\right)$.

Since $A S(B) \subseteq \operatorname{MonMod}(B)$, the above result implies that inductive equivalence in ISM does not require the condition of strong nor weak equivalence. It is worth noting 
that induction in ISM can be reformulated using classical logic. Given an NLP $B$, consider a clausal theory $C l(B)$ which is obtained from $B$ by replacing every NAF-literal not $A$ in (5) with a negative literal $\neg A$. Then, monotonic models of $B$ coincide with Herbrand models of $C l(B)$. Thus, the inductive equivalence in ISM is translated into the problem of inductive equivalence under the classical semantics, and Theorem 5.8 implies that $\operatorname{ISM}\left(B_{1}, E\right)=\operatorname{ISM}\left(B_{2}, E\right)$ iff $C l\left(B_{1}\right) \equiv C l\left(B_{2}\right)$.

\subsubsection{Brave induction from answer sets}

Sakama and Inoue (2009b) introduce an algorithm for brave induction in nonmonotonic logic programs. Given a background theory $B$ as an EDP and a set $O$ of ground literals as an observation, the algorithm BRAIN ${ }^{n o t}$ computes a set $H$ of rules as a hypothesis. Before presenting an algorithm, a couple of notions are in order. Given a literal $L, \operatorname{pred}(L)$ and const $(L)$ represent the predicate of $L$ and the constants appearing in $L$, respectively. Let $L_{0}$ be a ground literal and $S$ a set of ground literals. Then, $L_{1} \in S$ is relevant to $L_{0}$ if either (i) const $\left(L_{0}\right) \cap \operatorname{const}\left(L_{1}\right) \neq \emptyset$, or (ii) for some literal $L_{2} \in S$, const $\left(L_{1}\right) \cap \operatorname{const}\left(L_{2}\right) \neq \emptyset$ and $L_{2}$ is relevant to $L_{0}$. Otherwise, $L_{1} \in S$ is irrelevant to $L_{0}$. Rules $r_{1}, \ldots, r_{k}$ are comparable if there is a predicate appearing in every head $\left(r_{1}\right), \ldots$, head $\left(r_{k}\right)$.

BRAIN $^{\text {not }}$ constructs hypotheses in the following two steps. ${ }^{6}$ First, for a consistent answer set $S$ of $B$, construct a finite and consistent set $R_{S}$ of ground rules satisfying the following conditions. For any rule $r \in R_{S}$,

1. head $(r) \subseteq O$ and for any $L \in O$, there is a rule $r \in R_{S}$ such that head $(r)=\{L\}$,

2. $\operatorname{body}^{+}(r) \subseteq P$ where $P=\{L \mid L \in S$ and $L$ is relevant to the literal in head $(r)\}$,

3. $\operatorname{body}^{-}(r) \subseteq N$ where $N=\{L \mid L \in$ Lit $\backslash(S \cup \Theta)$ and $L$ is relevant to the literal in $\operatorname{head}(r)$ and appears in $\operatorname{ground}(B)\}$

where $\Theta=\{L \mid L \in$ Lit and $\operatorname{pred}(L)$ appears in $O\}$. In the second and third conditions, we put $\operatorname{body}^{+}(r)=P$ and $\operatorname{body}^{-}(r)=N$ if $P$ and $N$ are finite sets.

Second, for the set $R_{S}$ of rules obtained as above, $R_{S}$ is partitioned as $R_{S}=R_{1} \cup \cdots \cup R_{n}$ where each $R_{i}(1 \leq i \leq n)$ is a comparable set of ground rules. Then, the least generalization under subsumption of each $R_{i}$ is computed and collected as ${ }^{7}$

$$
\operatorname{lgs}\left(R_{S}\right)=\left\{\operatorname{lgs}\left(R_{1}\right), \ldots, \lg s\left(R_{n}\right)\right\}
$$

$\lg s\left(R_{S}\right)$ is a solution of brave induction if $B \cup \lg s\left(R_{S}\right)$ is consistent.

Example 5.4 Suppose the background theory $B$ :

$$
\begin{aligned}
& \operatorname{innocent}(x) \leftarrow \operatorname{not} \text { guilty }(x), \\
& \text { guilty }(x) \leftarrow \operatorname{not} \text { innocent }(x), \\
& \text { suspect }(a) \leftarrow, \\
& \text { suspect }(b) \leftarrow,
\end{aligned}
$$

\footnotetext{
${ }^{6}$ The algorithm in Sakama and Inoue (2009b) has additional two steps for constructing weak hypotheses and optimization, but we omit these steps here for simplicity reasons.

${ }^{7}$ The $\lg s$ of rules is computed in the same way as the case of clauses.
} 
which has four answer sets:

$$
\begin{aligned}
& S_{1}=\{\operatorname{suspect}(a), \operatorname{suspect}(b), \operatorname{guilty}(a), \operatorname{guilty}(b)\}, \\
& S_{2}=\{\operatorname{suspect}(a), \operatorname{suspect}(b), \operatorname{guilty}(a), \operatorname{innocent}(b)\}, \\
& S_{3}=\{\operatorname{suspect}(a), \operatorname{suspect}(b), \operatorname{innocent}(a), \operatorname{guilty}(b)\}, \\
& S_{4}=\{\operatorname{suspect}(a), \operatorname{suspect}(b), \operatorname{innocent}(a), \text { innocent }(b)\} .
\end{aligned}
$$

Given the observation $O=\{\operatorname{charged}(a)$, charged $(b)\}$, the set of ground rules

$$
\begin{array}{r}
R_{S_{1}}=\{\text { charged }(a) \leftarrow \operatorname{suspect}(a), \text { guilty }(a), \text { not innocent }(a), \\
\text { charged }(b) \leftarrow \operatorname{suspect}(b), \operatorname{guilty}(b), \text { not innocent }(b)\}
\end{array}
$$

is constructed using the answer set $S_{1}$. The $\operatorname{lgs}$ of $R_{S_{1}}$ becomes

$$
\operatorname{lgs}\left(R_{S_{1}}\right)=\{\operatorname{charged}(x) \leftarrow \operatorname{suspect}(x), \operatorname{guilty}(x), \operatorname{not} \text { innocent }(x)\},
$$

then $B \cup \operatorname{lgs}\left(R_{S_{1}}\right)$ has the answer set $S_{1} \cup\{\operatorname{charged}(a)$, charged $(b)\}$ which satisfies $O$.

By the definition, different hypotheses are constructed by different answer sets. Now we define inductive equivalence in $\mathrm{BRAIN}^{\text {not }}$ as follows.

Definition 5.3 (Inductive equivalence in BRAIN ${ }^{n o t}$ ) Two EDPs $B_{1}$ and $B_{2}$ are inductively equivalent in $\mathrm{BRAIN}^{n o t}$ if for any set $O$ of ground literals, $\lg s\left(R_{S}\right)=\lg s\left(R_{T}\right)$ holds for some consistent $S \in A S\left(B_{1}\right)$ and some consistent $T \in A S\left(B_{2}\right)$ such that $B_{1} \cup \lg s\left(R_{S}\right)$ and $B_{2} \cup \operatorname{lgs}\left(R_{T}\right)$ are consistent.

Then we have the following result.

Theorem 5.9 (Condition for inductive equivalence in BRAIN ${ }^{n o t}$ ) Two EDPs $B_{1}$ and $B_{2}$ are inductively equivalent in BRAIN ${ }^{\text {not }}$ iff $B_{1} \equiv_{s} B_{2}$.

Proof Suppose two ground programs $B_{1}$ and $B_{2}$ which are not strongly equivalent. Put $O=\{L\}$ such that $L$ appears nowhere in $B_{1}$, but $B_{2}$ contains the constraint $\leftarrow L$. With this setting, for some answer set $S$ of $B_{1}, R_{S}$ includes a rule $r$ such that head $(r)=L$, $\operatorname{body}^{+}(r) \subseteq S$ and $\operatorname{body}^{-}(r) \cap S=\emptyset$. Also, for some answer set $T$ of $B_{2}, R_{T}$ includes a rule $r$ such that $h e a d(r)=L, \operatorname{body}^{+}(r) \subseteq T$ and $\operatorname{body}^{-}(r) \cap T=\emptyset$. In this case, however, $B_{1} \cup R_{S}$ is consistent, but $B_{2} \cup R_{T}$ is inconsistent. Hence, $B_{1}$ and $B_{2}$ are not inductively equivalent. The converse implication clearly holds.

\section{Discussion}

\subsection{Comparison of conditions for inductive equivalence}

The results of this paper are summarized in Table 2. When the representation language is clausal logic, logical equivalence is necessary and sufficient for inductive equivalence between two background theories in each induction under both classical and the minimal 
Table 2 Comparison of conditions for inductive equivalence

\begin{tabular}{lll}
\hline Induction & \multicolumn{2}{l}{ Representation language (semantics) } \\
\cline { 2 - 3 } & Clausal logic (Mod, MM) & Nonmonotonic LP (AS) \\
\hline Caulnd & $B_{1} \equiv B_{2}$ & $B_{1} \equiv_{s} B_{2}$ \\
Bralnd & $B_{1} \equiv B_{2}$ & $B_{1} \equiv_{s} B_{2}$ \\
LFS & $B_{1} \equiv B_{2}$ & $B_{1} \equiv_{s} B_{2}$ \\
DesInd & $B_{1} \equiv B_{2}$ & $B_{1} \equiv_{s} B_{2}$ \\
\hline
\end{tabular}

model semantics. By contrast, when the representation language is nonmonotonic logic programming, strong equivalence is necessary and sufficient for inductive equivalence between two background theories in each induction under the answer set semantics. Since $B_{1} \equiv B_{2}$ iff $B_{1} \equiv_{s} B_{2}$ in clausal logic under both $\operatorname{SEM}(B)=\operatorname{Mod}(B)$ and $\operatorname{SEM}(B)=M M(B)$, we can conclude that strong equivalence of two background theories is necessary and sufficient for inductive equivalence in each induction. On the other hand, the condition of strong equivalence is sometimes relaxed to weak equivalence or other weaker equivalence relations in particular induction algorithms under restricted problem settings.

From the computational viewpoint, testing strong equivalence of propositional EDPs is converted to the problem of propositional entailment in classical logic (Lin 2002). The problem of testing strong equivalence is then solved using existing SAT solvers. For predicate programs with a finite domain, testing strong equivalence is also possible by instantiating a program into a finite propositional one. There is a system for testing strong equivalence of function-free finite nonmonotonic logic programs (Janhunen and Oikarinen 2004). Existence of no procedure for testing strong equivalence of logic programs with functions would restrict practical application of inductive equivalence in ILP. Nevertheless, inductive equivalence is efficiently testable when background theories are given as function-free finite Horn logic program (or Datalog) or a database that is a collection of propositional sentences.

\subsection{Relation to abductive equivalence}

Inoue and Sakama (2005, 2006a, 2006b) have studied equivalence relations in abductive frameworks. Given a background theory $B$ and a set $A$ of candidate hypotheses (called $a b$ ducibles), an abductive framework is defined as a tuple $\langle B, A\rangle$. Two abductive frameworks $\left\langle B_{1}, A_{1}\right\rangle$ and $\left\langle B_{2}, A_{2}\right\rangle$ are called explainable equivalent if, for any observation $O$, there is an explanation of $O$ in $\left\langle B_{1}, A_{1}\right\rangle$ iff there is an explanation of $O$ in $\left\langle B_{2}, A_{2}\right\rangle$. On the other hand, two programs are called explanatorily equivalent if, for any observation $O, O$ is an explanation of $O$ in $\left\langle B_{1}, A_{1}\right\rangle$ iff $O$ is an explanation of $O$ in $\left\langle B_{2}, A_{2}\right\rangle$. The former compares explainability of observations in different background theories, while the latter compares explanation contents of observations. Explanatory equivalence is stronger than explainable equivalence, and the former implies the latter. The paper (Inoue and Sakama 2005) introduces two equivalence notions for first-order abduction and abductive logic programming (ALP), and the paper (Inoue and Sakama 2006a) applies the notion to extended abduction of (Inoue and Sakama 1995). The paper (Inoue and Sakama 2006b) also argues equivalence between minimal explanations.

Comparing (Inoue and Sakama 2005, 2006a, 2006b) with our present work, some interesting connections are observed. When underlying logic is first-order logic, logical equivalence of two theories is a necessary and sufficient condition for explanatory equivalence in abduction. When a background theory is represented by a nonmonotonic logic program, on the other hand, $\left\langle B_{1}, A_{1}\right\rangle$ and $\left\langle B_{2}, A_{2}\right\rangle$ are explanatorily equivalent iff $B_{1}$ and $B_{2}$ are strongly 
equivalent. Those results have connection to the results of Theorems 3.3, 3.7, and 5.4 of this paper. However, there are some important differences between the previous studies on abductive equivalence and the results of this paper. First, the framework of ALP in Inoue and Sakama (2005, 2006a, 2006b) characterizes equivalence relations in brave abduction. That is, given a logic program $B$, a hypothesis $H$ explains an observation $G$ if $G$ is true in an answer set of $B \cup H$. This paper characterizes the problem of inductive equivalence not only in brave induction, but also in other forms of induction. Second, in abductive frameworks a hypothesis space $A$ is prespecified as abducibles and possible explanations for a given observation are constructed as a subset of abducibles. The existence of $A$ in abductive logic programs results in characterization by relative strong equivalence, i.e., two programs $B_{1}$ and $B_{2}$ are explanatory equivalent iff they are strongly equivalent with respect to $A$. Moreover, in abductive logic programming, abducibles and observations are usually restricted to (ground) literals. In ILP, on the other hand, hypotheses and observations are general rules rather than facts. Besides these differences, both abduction and induction require strong equivalence of two (nonmonotonic) logic programs to identify the results of abductive/inductive inference. The essence of this lies in the fact that abduction and induction are both ampliative reasoning and extend theories. Strong equivalence takes the influence of addition of a rule set to each program into account, so that it succeeds in characterizing the effect of abduction/induction that are not captured by weak equivalence of programs. In Lifschitz et al. (2001), it is argued that strong equivalence is useful to simplify a part of a program without looking at the other parts. On the other hand, a series of studies (Inoue and Sakama 2005, 2006a, 2006b) and the result of this paper reveal that strong equivalence has another important applications for testing equivalence of background theories in abductive and inductive logic programming.

\subsection{Program development in ILP}

As presented in Sect. 4, there are many ILP systems which handle Horn logic programs as background theories. In Horn logic programs, program transformations which preserve weak equivalence of programs are popularly used for optimizing programs. Partial evaluation or unfold/fold transformations are of this kind (Tamaki and Sato 1984; Pettorossi and Proietti 1994). For instance, given the program

$$
B_{1}=\{p(x) \leftarrow q(x), \quad q(x) \leftarrow r(x), \quad r(a) \leftarrow\},
$$

unfolding the first clause by the second one results in the program

$$
B_{2}=\{p(x) \leftarrow r(x), \quad q(x) \leftarrow r(x), \quad r(a) \leftarrow\} .
$$

On the other hand, in $B_{2}$ folding the first clause by the second one results in the program $B_{1}$. $B_{1}$ and $B_{2}$ have the same least model thereby weakly equivalent, but not logically equivalent. In ILP, unfolding is often used as an operator for specialization (Boström and IdestamAlmquist 1994), and folding is used as an operator for generalization under the name of inverse resolution (Muggleton and Buntine 1992).

In Sect. 3.2 we observe that logical equivalence of two clausal theories is necessary and sufficient to guarantee inductive equivalence under the minimal model semantics. Since weak equivalence provides a weaker condition than logical equivalence (Proposition 2.3), the condition of weak equivalence of two clausal theories is not sufficient for preserving inductive equivalence under the minimal model semantics in general. This result brings 
an important implication in program development in ILP that basic program transformations, such as unfold/fold transformations, are not applicable for optimizing background theories in ILP. If used, those transformations change solutions of induction in general. In the above example, $B_{1}$ and $B_{2}$ are not inductively equivalent in explanatory induction as $H=\{q(a) \leftarrow\}$ explains $p(a)$ in $B_{1}$ but does not in $B_{2}$. Nevertheless, those transformations are still effective as far as one uses induction algorithms that require the condition of weak equivalence. In Sect. 4, we observe that FOIL and GOLEM are of this kind, but PROGOL and CLAUDIEN are not. It is also known that unfold/fold transformations do not preserve strong equivalence of nonmonotonic logic programs (Osorio et al. 2001), so that those transformations cannot be used for program optimization in nonmonotonic ILP without changing solutions in general.

\subsection{Verification of algorithms}

If an induction algorithm produces different hypotheses from two different background theories, those theories are considered to be inductively inequivalent. It may happen, however, that some algorithm may produce different hypotheses from two background theories due to its incompleteness/incorrectness. If two strongly equivalent programs induce different hypotheses in face of some observation, it indicates that the induction algorithm is incomplete or incorrect. In this way, inductive equivalence would be used for testing correctness/completeness of induction algorithms. We consider that any induction algorithm should compute the same hypotheses from two different background theories as far as they are inductively equivalent. With this regard, inductive equivalence has an application to verification of induction algorithms.

For another application, inductive equivalence would be used for comparing capabilities of different induction algorithms. Let $\alpha(B, O)$ be the set of hypotheses induced by an algorithm $\alpha$ using a background theory $B$ and an observation $O$. For two different induction algorithms $\alpha_{1}$ and $\alpha_{2}$ under a common problem setting, suppose that $\alpha_{1}\left(B_{1}, O\right)=\alpha_{1}\left(B_{2}, O\right)$ implies $\alpha_{2}\left(B_{1}, O\right)=\alpha_{2}\left(B_{2}, O\right)$, but not vice versa. In this case, $\alpha_{1}$ is considered inductively more sensitive than $\alpha_{2}$ in the sense that $\alpha_{1}$ may distinguish different background theories that are not distinguished by $\alpha_{2}$. For instance, suppose any ground Horn logic program $B$ and any set $O$ of ground atoms. In this problem setting, we can say that PROGOL is inductively more sensitive than GoLEM, since $\operatorname{bot}\left(B_{1}, E\right)=\operatorname{bot}\left(B_{2}, O\right)$ implies $\operatorname{rlgs}\left(M_{B_{1}}, O\right)=\operatorname{rlgs}\left(M_{B_{2}}, O\right)$ but not vice versa. (This is due to the fact that $B_{1} \equiv B_{2}$ implies $B_{1} \equiv_{w} B_{2}$ but not vice versa.) Thus, inductive equivalence is also useful for evaluating capabilities of induction algorithms.

\section{Concluding remarks}

This paper has studied equivalence issues in induction and inductive logic programming. We introduced the notion of inductive equivalence which compares hypotheses that explain observations with respect to different background theories. Two different logics for representation languages - clausal theories and nonmonotonic logic programming, and four different frameworks of induction - cautious induction, brave induction, learning from satisfiability, and descriptive induction, were considered. The results of this paper show that logical equivalence is necessary and sufficient for inductive equivalence in clausal theories, while strong equivalence is necessary and sufficient in nonmonotonic extended disjunctive programs. On the other hand, we also observed that existing Horn ILP systems sometimes 
require weaker conditions of equivalence under restricted problem settings. We addressed that inductive equivalence has potential applications for verification and evaluation of induction algorithms. We also argued that program transformations which are popularly used in logic programming generally do not preserve inductive equivalence of programs. This is an important caution for program development in ILP which has been receiving little attention in the field.

Inductive equivalence considered in this paper guarantees coincidence of every hypothesis induced by different background theories. In practice, however, the exact coincidence of whole hypotheses is not always requested and one may be interested in preserving some preferred hypotheses. The criteria of preference of hypothesis depends on applications and it is often specified under the name of induction bias. In the context of abduction, preferred hypotheses are referred to "best explanations". In Inoue and Sakama (2006b), it is proved that two abductive theories are explanatory equivalent iff they have the same minimal explanations for any observation. Sakama and Inoue (1995) introduce several program transformations which preserve best explanations in abductive logic programming. Inductive equivalence of preferred hypotheses and program transformations for preserving those hypotheses are left for future research.

\section{References}

Badea, L., \& Stanciu, M. (1999). Refinement operators can be (weakly) perfect. In Lecture notes in artificial intelligence: Vol. 1634. Proceedings of the 9th international workshop on inductive logic programming (pp. 21-32). Berlin: Springer.

Baral, C., \& Gelfond, M. (1994). Logic programming and knowledge representation. Journal of Logic Programming, 19/20, 73-148.

Bossu, G., \& Siegel, P. (1985). Saturation, nonmonotonic reasoning and the closed-world assumption. Artificial Intelligence, 25, 13-63.

Boström, H., \& Idestam-Almquist, P. (1994) Specialization of logic programs by pruning SLD-trees. In Proceedings of the 4th international workshop on inductive logic programming (pp. 31-48).

Clark, K. L. (1978). Negation as failure. In H. Gallaire, \& J. Minker (Eds.), Logic and data bases (pp. 293322). New York: Plenum.

De Raedt, L. (1997). Logical settings for concept-learning. Artificial Intelligence, 95, 187-201.

De Raedt, L., \& Bruynooghe, M. (1993). A theory of clausal discovery. In Proceedings of the 13th international joint conference on artificial intelligence (pp. 1058-1063). San Mateo: Morgan Kaufmann.

De Raedt, L., \& Dehaspe, L. (1997a) Learning from satisfiability. In Proceedings of the 9th Dutch conference on artificial intelligence (pp. 303-312).

De Raedt, L., \& Dehaspe, L. (1997b). Clausal discovery. Machine Learning, 26(2-3), 99-146.

De Raedt, L., \& Lavrač, N. (1993). The many faces of inductive logic programming. In Lecture notes in computer science: Vol. 689. Methodologies for intelligent systems, 7th international symposium (pp. 435449). Berlin: Springer.

Denecker, M., \& Kakas, A. C. (2002). Abductive logic programming. In A. C. Kakas, \& F. Sadri (Eds.), Lecture notes in artificial intelligence: Vol. 2407. Computational logic: logic programming and beyondessays in honour of Robert A. Kowalski, Part I (pp. 402-436). Berlin: Springer.

Eiter, T., \& Fink, M. (2003). Uniform equivalence of logic programs under the stable model semantics. In Lecture notes in computer sciences: Vol. 2916. Proceedings of the 19th international conference on logic programming (pp. 224-238). Berlin: Springer.

Flach, P. A. (1996). Rationality postulates for induction. In Proceedings of the 6th international conference on theoretical aspects of rationality and knowledge (pp. 267-281). San Mateo: Morgan Kaufmann.

Flach, P. A., \& Kakas, A. C. (2000). Abductive and inductive reasoning: background and issues. In P. A. Flach, \& A. C. Kakas (Eds.), Abduction and induction-essays on their relation and integration (pp. 1-27). Norwell: Kluwer Academic.

Gelfond, M., \& Lifschitz, V. (1988). The stable model semantics for logic programming. In Proceedings of the 5th international conference and symposium on logic programming (pp. 1070-1080). Cambridge: MIT Press. 
Gelfond, M., \& Lifschitz, V. (1991). Classical negation in logic programs and disjunctive databases. New Generation Computing, 9, 365-385.

Inoue, K. (2004). Induction as consequent finding. Machine Learning, 55, 109-135.

Inoue, K., \& Sakama, C. (1995). Abductive framework for nonmonotonic theory change. In Proceedings of the 14th international joint conference on artificial intelligence (pp. 204-210). San Mateo: Morgan Kaufmann.

Inoue, K., \& Sakama, C. (2004). Equivalence of logic programs under updates. In Lecture notes in artificial intelligence: Vol. 3229. Proceedings of the 9th European conference on logics in artificial intelligence (pp. 174-186). Berlin: Springer.

Inoue, K., \& Sakama, C. (2005) Equivalence in abductive logic. In Proceedings of the 19th international joint conference on artificial intelligence (pp. 472-477).

Inoue, K., \& Sakama, C. (2006a). On abductive equivalence. In L. Magnani (Ed.), Model-based reasoning in science and engineering: cognitive science, epistemology, logic. Studies in logic (pp. 333-352). London: College Publications.

Inoue, K., \& Sakama, C. (2006b). Abductive equivalence in first-order logic. Logic Journal of the IGPL. Special Issue: Abduction, Practical Reasoning, and Creative Inferences in Science, 14(2), 333-346.

Janhunen, T., \& Oikarinen, E. (2004). LPEQ and DLPEQ - translators for automated equivalence testing of logic programs. In Lecture notes in artificial intelligence: Vol. 2923. Proceedings of the 7th international conference of logic programming and nonmonotonic reasoning (pp. 336-340). Berlin: Springer.

Lachiche, N. (2000). Abduction and induction from a non-monotonic reasoning perspective. In P. A. Flach, \& A. C. Kakas (Eds.), Abduction and induction-essays on their relation and integration (pp. 107-116). Norwell: Kluwer Academic.

Lifschitz, V., Pearce, D., \& Valverde, A. (2001). Strongly equivalent logic programs. ACM Transactions on Computational Logic, 2, 526-541.

Lin, F. (2002). Reducing strong equivalence of logic programs to entailment in classical propositional logic. In Proceedings of the 8th international conference on principles of knowledge representation and reasoning (pp. 170-176). San Mateo: Morgan Kaufmann.

Maher, M. J. (1988). Equivalence of logic programs. In J. Minker (Ed.), Foundations of deductive databases and logic programming (pp. 627-658). San Mateo: Morgan Kaufmann.

McCarthy, J. (1980). Circumscription-a form of nonmonotonic reasoning. Artificial Intelligence, 13, $27-39$.

Minker, J. (1982). On indefinite data bases and the closed world assumption. In Lecture notes in computer science: Vol. 138. Proceedings of the 6th international conference on automated deduction (pp. 292308). Berlin: Springer.

Muggleton, S. (Ed.) (1992). Inductive logic programming. San Diego: Academic Press.

Muggleton, S. (1995). Inverse entailment and progol. New Generation Computing, 13, 245-286.

Muggleton, S., \& Buntine, W. (1992). Machine invention of first-order predicate by inverting resolution. In S. Muggleton (Ed.), Inductive logic programming (pp. 261-280). San Diego: Academic Press.

Muggleton, S., \& Feng, C. (1990). Efficient induction algorithm. In S. Muggleton (Ed.), Inductive logic programming (pp. 281-298). San Diego: Academic Press.

Nienhuys-Cheng, S.-H., \& De Wolf, R. (1997). Lecture notes in artificial intelligence: Vol. 228. Foundations of inductive logic programming. Berlin: Springer.

Osorio, M., Navarro, J. A., \& Arrazola, J. (2001). Equivalence in answer set programming. In Lecture notes in computer science: Vol. 2372. Proceedings of the 11th international workshop on logic based program synthesis and transformation (pp. 57-75). Berlin: Springer.

Otero, R. P. (2001). Induction of stable models. In Lecture notes in artificial intelligence: Vol. 2157. Proceedings of the 11th international conference on inductive logic programming (pp. 193-205). Berlin: Springer.

Pettorossi, A., \& Proietti, M. (1994). Transformation of logic programs: foundations and techniques. Journal of Logic Programming, 19/20, 261-320.

Plotkin, G. D. (1971). A further note on inductive generalization. In B. Meltzer, \& D. Michie (Eds.), Machine intelligence (Vol. 6, pp. 101-124). Edinburgh: Edinburgh University Press.

Quinlan, R. (1990). Learning logical definitions from relations. Machine Learning, 5, 239-266.

Sagiv, Y. (1988). Optimizing datalog programs. In J. Minker (Ed.), Foundations of deductive databases and logic programming (pp. 659-668). San Mateo: Morgan Kaufmann.

Sakama, C., \& Inoue, K. (1995). The effect of partial deduction in abductive reasoning. In Proceedings of the 12th international conference on logic programming (pp. 383-397). Cambridge: MIT Press.

Sakama, C., \& Inoue, K. (2005). Inductive equivalence of logic programs. In Lecture notes in artificial intelligence: Vol. 3625. Proceedings of the 15th international conference on inductive logic programming (pp. 312-329). Berlin: Springer.

Sakama, C., \& Inoue, K. (2009a). Equivalence issues in abduction and induction. Journal of Applied Logic, 7(3), 318-328. 
Sakama, C., \& Inoue, K. (2009b). Brave induction: a logical framework for learning from incomplete information. Machine Learning, 76(1), 3-35.

Tamaki, H., \& Sato, T. (1984). Unfold/fold transformation of logic programs. In Proceedings of the 2nd international conference on logic programming (pp. 127-138).

Turner, H. (2003). Strong equivalence made easy: nested expressions and weight constraints. Theory and Practice of Logic Programming, 3(4-5), 609-622.

Van Emden, M. H., \& Kowalski, R. A. (1976). The semantics of predicate logic as a programming language. Journal of the ACM, 23(4), 733-742. 